\title{
Ubiquitous production of branched glycerol dialkyl glycerol tetraethers (brGDGTs) in global marine environments: a new source indicator for brGDGTs
}

\section{Wenjie Xiao et al.}

Correspondence to: Yunping Xu (ypxu@shou.edu.cn)

The copyright of individual parts of the supplement might differ from the CC-BY 3.0 licence. 


\section{M1 CORE}

$\begin{array}{lllll}\text { Sample } & \text { Latitude } & \text { Longitude } & \text { IIIa/IIa } & \text { BIT } \\ \text { BH1 2-3 } & 37.52 & 119.32 & 0.60 & 0.41 \\ \text { BH1 3-4 } & 37.52 & 119.32 & 0.55 & 0.42 \\ \text { BH1 5-6 } & 37.52 & 119.32 & 0.59 & 0.50 \\ \text { BH1 6-7 } & 37.52 & 119.32 & 0.61 & 0.55 \\ \text { BH1 7-8 } & 37.52 & 119.32 & 0.62 & 0.53 \\ \text { BH1 10-11 } & 37.52 & 119.32 & 0.68 & 0.74 \\ \text { BH1 12-13 } & 37.52 & 119.32 & 0.63 & 0.75 \\ \text { BH1 14-15 } & 37.52 & 119.32 & 0.60 & 0.76 \\ \text { BH1 16-17 } & 37.52 & 119.32 & 0.65 & 0.73 \\ \text { BH1 17-18 } & 37.52 & 119.32 & 0.57 & 0.76 \\ \text { BH1 19-20 } & 37.52 & 119.32 & 0.60 & 0.73 \\ \text { BH1 21-22 } & 37.52 & 119.32 & 0.68 & 0.70 \\ \text { BH1 23-24 } & 37.52 & 119.32 & 0.72 & 0.74 \\ \text { BH1 25-26 } & 37.52 & 119.32 & 0.61 & 0.74 \\ \text { BH1 26-27 } & 37.52 & 119.32 & 0.68 & 0.73 \\ \text { BH1 28-29 } & 37.52 & 119.32 & 0.59 & 0.74 \\ \text { BH1 30-31 } & 37.52 & 119.32 & 0.68 & 0.49 \\ \text { BH1 33-34 } & 37.52 & 119.32 & 0.67 & 0.33 \\ \text { BH1 35-36 } & 37.52 & 119.32 & 0.66 & 0.28 \\ \text { BH1 37-38 } & 37.52 & 119.32 & 0.67 & 0.27 \\ \text { BH1 39-40 } & 37.52 & 119.32 & 0.59 & 0.28 \\ \text { BH1 41-42 } & 37.52 & 119.32 & 0.60 & 0.30 \\ \text { BH1 43-44 } & 37.52 & 119.32 & 0.58 & 0.29 \\ \text { BH1 45-46 } & 37.52 & 119.32 & 0.58 & 0.31 \\ \text { BH1 47-48 } & 37.52 & 119.32 & 0.59 & 0.30 \\ \text { BH1 49-50 } & 37.52 & 119.32 & 0.57 & 0.31 \\ \text { BH1 51-52 } & 37.52 & 119.32 & 0.54 & 0.34 \\ \text { BH1 55-56 } & 37.52 & 119.32 & 0.62 & 0.37 \\ \text { BH1 57-58 } & 37.52 & 119.32 & 0.64 & 0.40 \\ \text { BH1 59-60 } & 37.52 & 119.32 & 0.70 & 0.38 \\ \text { BH1 61-62 } & 37.52 & 119.32 & 0.62 & 0.36 \\ \text { BH1 63-64 } & 37.52 & 119.32 & 0.64 & 0.31\end{array}$

\section{M3 CORE}

$\begin{array}{lllll}\text { M3 0-1 } & 38.66 & 119.54 & 1.01 & 0.04 \\ \text { M3 1-2 } & 38.66 & 119.54 & 1.16 & 0.04 \\ \text { M3 2-3 } & 38.66 & 119.54 & 1.16 & 0.04 \\ \text { M3 3-4 } & 38.66 & 119.54 & 1.12 & 0.04 \\ \text { M3 4-5 } & 38.66 & 119.54 & 1.21 & 0.04 \\ \text { M3 5-6 } & 38.66 & 119.54 & 1.11 & 0.04 \\ \text { M3 6-7 } & 38.66 & 119.54 & 1.18 & 0.04 \\ \text { M3 7-8 } & 38.66 & 119.54 & 1.19 & 0.05 \\ \text { M3 8-9 } & 38.66 & 119.54 & 1.08 & 0.05 \\ \text { M3 9-10 } & 38.66 & 119.54 & 1.14 & 0.06 \\ \text { M3 10-11 } & 38.66 & 119.54 & 1.11 & 0.07 \\ \text { M3 11-12 } & 38.66 & 119.54 & 1.03 & 0.08 \\ \text { M3 12-13 } & 38.66 & 119.54 & 1.08 & 0.07 \\ \text { M3 13-14 } & 38.66 & 119.54 & 1.14 & 0.08 \\ \text { M3 14-15 } & 38.66 & 119.54 & 1.10 & 0.08 \\ \text { M3 15-16 } & 38.66 & 119.54 & 1.12 & 0.08 \\ \text { M3 16-17 } & 38.66 & 119.54 & 1.17 & 0.08 \\ \text { M3 17-18 } & 38.66 & 119.54 & 1.29 & 0.10\end{array}$




\begin{tabular}{|c|c|c|c|c|}
\hline M3 18-19 & 38.66 & 119.54 & 1.26 & 0.11 \\
\hline M3 19-20 & 38.66 & 119.54 & 1.23 & 0.11 \\
\hline M3 20-21 & 38.66 & 119.54 & 1.31 & 0.12 \\
\hline M3 21-22 & 38.66 & 119.54 & 1.22 & 0.13 \\
\hline M3 22-23 & 38.66 & 119.54 & 1.25 & 0.13 \\
\hline M3 23-24 & 38.66 & 119.54 & 1.26 & 0.12 \\
\hline M3 24-25 & 38.66 & 119.54 & 1.29 & 0.11 \\
\hline M3 25-26 & 38.66 & 119.54 & 1.25 & 0.10 \\
\hline M3 26-27 & 38.66 & 119.54 & 1.48 & 0.14 \\
\hline M3 27-28 & 38.66 & 119.54 & 1.36 & 0.13 \\
\hline M3 28-29 & 38.66 & 119.54 & 1.24 & 0.12 \\
\hline M3 29-30 & 38.66 & 119.54 & 1.31 & 0.13 \\
\hline M3 30-31 & 38.66 & 119.54 & 1.29 & 0.13 \\
\hline M3 31-32 & 38.66 & 119.54 & 1.26 & 0.15 \\
\hline M3 32-33 & 38.66 & 119.54 & 1.41 & 0.16 \\
\hline M3 33-34 & 38.66 & 119.54 & 1.49 & 0.15 \\
\hline M3 34-35 & 38.66 & 119.54 & 1.33 & 0.16 \\
\hline M3 35-36 & 38.66 & 119.54 & 1.39 & 0.15 \\
\hline M3 36-37 & 38.66 & 119.54 & 1.29 & 0.15 \\
\hline M3 37-38 & 38.66 & 119.54 & 1.41 & 0.18 \\
\hline M3 38-39 & 38.66 & 119.54 & 1.39 & 0.17 \\
\hline M3 39-40 & 38.66 & 119.54 & 1.33 & 0.18 \\
\hline M3 40-41 & 38.66 & 119.54 & 1.42 & 0.15 \\
\hline M3 41-42 & 38.66 & 119.54 & 1.41 & 0.16 \\
\hline M3 42-43 & 38.66 & 119.54 & 1.46 & 0.14 \\
\hline M3 43-44 & 38.66 & 119.54 & 1.43 & 0.16 \\
\hline M3 44-45 & 38.66 & 119.54 & 1.40 & 0.19 \\
\hline M3 45-46 & 38.66 & 119.54 & 1.51 & 0.18 \\
\hline M3 46-47 & 38.66 & 119.54 & 1.41 & 0.18 \\
\hline M3 47-48 & 38.66 & 119.54 & 1.53 & 0.21 \\
\hline M3 48-49 & 38.66 & 119.54 & 1.47 & 0.24 \\
\hline M3 49-50 & 38.66 & 119.54 & 1.40 & 0.22 \\
\hline M3 50-51 & 38.66 & 119.54 & 1.50 & 0.24 \\
\hline M3 51-52 & 38.66 & 119.54 & 1.40 & 0.22 \\
\hline M3 52-53 & 38.66 & 119.54 & 1.41 & 0.23 \\
\hline M3 53-54 & 38.66 & 119.54 & 1.42 & 0.22 \\
\hline M3 54-55 & 38.66 & 119.54 & 1.42 & 0.22 \\
\hline M3 55-56 & 38.66 & 119.54 & 1.42 & 0.23 \\
\hline M3 56-57 & 38.66 & 119.54 & 1.50 & 0.22 \\
\hline M3 57-58 & 38.66 & 119.54 & 1.45 & 0.25 \\
\hline M3 58-59 & 38.66 & 119.54 & 1.45 & 0.23 \\
\hline M3 59-60 & 38.66 & 119.54 & 1.52 & 0.24 \\
\hline M3 54-55 & 38.66 & 119.54 & 1.45 & 0.22 \\
\hline M3 54-55 & 38.66 & 119.54 & 1.42 & 0.22 \\
\hline M3 45-46 & 38.66 & 119.54 & 1.54 & 0.21 \\
\hline M3 15-16 & 38.66 & 119.54 & 1.16 & 0.08 \\
\hline \multicolumn{5}{|l|}{ M7 CORE } \\
\hline M7 0-1 & 39.53 & 120.46 & 1.06 & 0.04 \\
\hline M7 1-2 & 39.53 & 120.46 & 0.94 & 0.05 \\
\hline M7 2-3 & 39.53 & 120.46 & 0.95 & 0.05 \\
\hline M7 3-4 & 39.53 & 120.46 & 0.95 & 0.05 \\
\hline M7 4-5 & 39.53 & 120.46 & 0.92 & 0.05 \\
\hline M7 5-6 & 39.53 & 120.46 & 0.95 & 0.05 \\
\hline
\end{tabular}




\begin{tabular}{|c|c|c|c|c|}
\hline M7 6-7 & 39.53 & 120.46 & 0.98 & 0.06 \\
\hline M7 9-10 & 39.53 & 120.46 & 0.98 & 0.07 \\
\hline M7 12-13 & 39.53 & 120.46 & 1.05 & 0.08 \\
\hline M7 13-14 & 39.53 & 120.46 & 1.04 & 0.09 \\
\hline M7 14-15 & 39.53 & 120.46 & 1.07 & 0.08 \\
\hline M7 17-18 & 39.53 & 120.46 & 1.19 & 0.12 \\
\hline M7 18-19 & 39.53 & 120.46 & 1.09 & 0.11 \\
\hline M7 19-20 & 39.53 & 120.46 & 1.03 & 0.11 \\
\hline M7 20-21 & 39.53 & 120.46 & 1.02 & 0.12 \\
\hline M7 21-22 & 39.53 & 120.46 & 0.98 & 0.12 \\
\hline M7 22-23 & 39.53 & 120.46 & 1.02 & 0.11 \\
\hline M7 23-24 & 39.53 & 120.46 & 1.06 & 0.11 \\
\hline M7 24-25 & 39.53 & 120.46 & 1.04 & 0.11 \\
\hline M7 25-26 & 39.53 & 120.46 & 1.05 & 0.12 \\
\hline M7 26-27 & 39.53 & 120.46 & 1.11 & 0.11 \\
\hline M7 27-28 & 39.53 & 120.46 & 1.26 & 0.12 \\
\hline M7 29-30 & 39.53 & 120.46 & 1.13 & 0.11 \\
\hline M7 30-31 & 39.53 & 120.46 & 1.16 & 0.09 \\
\hline M7 31-32 & 39.53 & 120.46 & 1.11 & 0.12 \\
\hline M7 32-33 & 39.53 & 120.46 & 1.10 & 0.12 \\
\hline M7 33-34 & 39.53 & 120.46 & 1.09 & 0.10 \\
\hline M7 34-35 & 39.53 & 120.46 & 1.07 & 0.11 \\
\hline M7 35-36 & 39.53 & 120.46 & 0.98 & 0.11 \\
\hline M7 36-37 & 39.53 & 120.46 & 1.04 & 0.12 \\
\hline M7 37-38 & 39.53 & 120.46 & 1.12 & 0.13 \\
\hline M7 38-39 & 39.53 & 120.46 & 1.07 & 0.12 \\
\hline M7 39-40 & 39.53 & 120.46 & 1.01 & 0.13 \\
\hline M7 40-41 & 39.53 & 120.46 & 1.05 & 0.14 \\
\hline M7 41-42 & 39.53 & 120.46 & 1.08 & 0.12 \\
\hline M7 42-43 & 39.53 & 120.46 & 1.06 & 0.14 \\
\hline M7 43-44 & 39.53 & 120.46 & 1.07 & 0.12 \\
\hline M7 45-46 & 39.53 & 120.46 & 1.05 & 0.13 \\
\hline M7 46-47 & 39.53 & 120.46 & 1.01 & 0.11 \\
\hline M7 47-48 & 39.53 & 120.46 & 1.16 & 0.12 \\
\hline M7 48-49 & 39.53 & 120.46 & 1.12 & 0.13 \\
\hline M7 49-50 & 39.53 & 120.46 & 0.98 & 0.13 \\
\hline M7 51-52 & 39.53 & 120.46 & 1.00 & 0.16 \\
\hline M7 52-53 & 39.53 & 120.46 & 0.95 & 0.18 \\
\hline
\end{tabular}

Archaeal tetraether lipids record subsurface water temperature in the South China Sea http://dx.doi.org/10

$\begin{array}{lllll}17941 & 118.48 & 21.52 & 0.72 & 0.14 \\ 17942 & 113.20 & 19.33 & 0.85 & 0.07 \\ 17943 & 117.55 & 18.95 & 1.38 & 0.11 \\ 17944 & 113.64 & 18.66 & 1.27 & 0.08 \\ 17945 & 113.78 & 18.13 & 1.13 & 0.05 \\ 17946 & 114.25 & 18.13 & 1.65 & 0.22 \\ 17947 & 116.03 & 18.47 & 1.40 & 0.07 \\ 17948 & 114.90 & 16.71 & 1.46 & 0.25 \\ 17949 & 115.17 & 17.35 & 1.72 & 0.15 \\ 17950 & 112.90 & 16.09 & 1.19 & 0.08 \\ 17951 & 113.41 & 16.29 & 0.96 & 0.09 \\ 17952 & 114.47 & 16.67 & 1.60 & 0.11 \\ 17954 & 111.53 & 14.80 & 1.09 & 0.10 \\ 17955 & 112.18 & 14.12 & 1.26 & 0.14\end{array}$




$\begin{array}{lrlll}17956 & 112.59 & 13.85 & 1.55 & 0.25 \\ 17957 & 115.30 & 10.90 & 2.48 & 0.06 \\ 17958 & 115.08 & 11.62 & 1.59 & 0.09 \\ 17959 & 115.29 & 11.14 & 1.95 & 0.03 \\ 17960 & 115.56 & 10.12 & 2.36 & 0.06 \\ 17962 & 112.08 & 7.18 & 0.80 & 0.05 \\ 17963 & 112.67 & 6.17 & 0.78 & 0.08 \\ 17964 & 112.21 & 6.16 & 0.68 & 0.05 \\ 17965 & 112.55 & 6.16 & 0.83 & 0.08 \\ \text { S031 } & 111.00 & 10.50 & 1.20 & 0.13 \\ \text { S052 } & 115.50 & 7.25 & 3.59 & 0.09 \\ 199 & 118.93 & 16.17 & 1.19 & 0.11 \\ \text { A231-X15 } & 119.50 & 21.25 & 0.84 & 0.10 \\ \text { A290-17 } & 119.50 & 20.25 & 1.08 & 0.09 \\ \text { A296-98 } & 119.88 & 19.31 & 0.64 & 0.23 \\ \text { A380-137 } & 118.36 & 17.28 & 1.38 & 0.23 \\ \text { A381-181 } & 117.84 & 17.29 & 4.14 & 0.13 \\ 126-03-07-72119.09 & 20.65 & 0.99 & 0.22\end{array}$

Organic-geochemical proxies of sea surface temperature in surface sediments of the tropical eastern Indian ।

$\begin{array}{lllll}10008-4 & -0.95 & 98.25 & 1.62 & 0.05 \\ 10010-1 & -1.02 & 97.08 & 1.14 & 0.08 \\ 10014-1 & 1.67 & 96.97 & 1.43 & 0.07 \\ 10015-1 & 1.62 & 96.88 & 1.52 & 0.06 \\ 10016-2 & 1.58 & 96.65 & 1.57 & 0.06 \\ 10022-3 & -0.48 & 98.85 & 1.22 & 0.04 \\ 10024-3 & -0.77 & 99.27 & 1.74 & 0.07 \\ 10025-3 & -0.67 & 99.12 & 1.18 & 0.05 \\ 10026-2 & -0.93 & 99.52 & 1.42 & 0.04 \\ 10027-3 & -0.80 & 99.65 & 1.52 & 0.06 \\ 10028-4 & -0.68 & 99.75 & 1.18 & 0.04 \\ 10029-3 & -1.48 & 100.12 & 1.03 & 0.04 \\ 10031-3 & -1.68 & 99.60 & 1.36 & 0.06 \\ 10032-1 & -1.67 & 99.67 & 1.29 & 0.07 \\ 10033-5 & -1.55 & 99.95 & 1.29 & 0.04 \\ 10034-3 & -4.15 & 101.48 & 1.43 & 0.06 \\ 10036-3 & -5.33 & 103.65 & 0.41 & 0.22 \\ 10037-2 & -5.47 & 103.55 & 0.37 & 0.19 \\ 10038-3 & -5.93 & 103.23 & 0.81 & 0.14 \\ 10039-3 & -5.87 & 103.28 & 1.05 & 0.06 \\ 10040-3 & -6.47 & 102.85 & 1.01 & 0.04 \\ 10041-3 & -6.27 & 103.00 & 1.75 & 0.04 \\ 10043-2 & -7.30 & 105.05 & 0.77 & 0.07 \\ 10044-3 & -8.48 & 109.00 & 0.94 & 0.13 \\ 10047-1 & -9.30 & 109.00 & 1.26 & 0.04 \\ 10049-5 & -8.78 & 110.48 & 0.91 & 0.06 \\ 10050-1 & -9.45 & 110.43 & 1.06 & 0.02 \\ 10058-1 & -8.68 & 112.63 & 0.82 & 0.05 \\ 10059-1 & -8.67 & 112.87 & 0.70 & 0.05 \\ 10061-5 & -9.72 & 113.02 & 1.52 & 0.03 \\ 10063-5 & -9.63 & 118.15 & 0.48 & 0.01 \\ 10064-5 & -9.53 & 118.30 & 0.61 & 0.01 \\ 10066-6 & -9.38 & 118.57 & 1.06 & 0.02 \\ 10067-5 & -9.13 & 119.28 & 1.02 & 0.03\end{array}$


$\begin{array}{lllll}10068-1 & -9.58 & 121.15 & 0.99 & 0.02 \\ 10069-4 & -9.58 & 120.90 & 1.28 & 0.01\end{array}$

A re-evaluation of the use of branched GDGTs as terrestrial biomarkers: Implications for the BIT Index htt

$\begin{array}{lllll}\text { STN-1 } & -168.08 & 67.03 & 0.83 & 0.13 \\ \text { WHS-2 } & -167.03 & 70.05 & 1.50 & 0.05 \\ \text { WHS-5 } & -160.05 & 72.08 & 2.00 & 0.03 \\ \text { WHS-6 } & -160.00 & 73.02 & & \\ \text { WHS-7 } & -159.07 & 73.03 & 1.40 & 0.09 \\ \text { WHS-12 } & -159.05 & 73.05 & 1.00 & 0.46 \\ \text { EHS-4 } & -157.08 & 73.08 & 2.00 & 0.05 \\ \text { EHS-6 } & -158.07 & 72.05 & 1.67 & 0.03 \\ \text { EHS-9 } & -158.03 & 72.08 & 1.40 & 0.04 \\ \text { EHS-11 } & -158.00 & 73.00 & 1.27 & 0.05 \\ \text { EHS-12 } & -157.05 & 73.03 & & 0.13 \\ \text { BC-3 } & -156.07 & 73.07 & 0.95 & 0.18 \\ \text { BC-4 } & -155.07 & 71.05 & 0.88 & 0.11 \\ \text { BC-5 } & -154.07 & 71.08 & 0.80 & 0.12 \\ \text { BC-7 } & -154.03 & 72.00 & 1.00 & 0.04 \\ \text { EB-2 } & -154.03 & 72.05 & 0.86 & 0.19 \\ \text { EB-4 } & -152.05 & 71.03 & 0.88 & 0.28 \\ \text { EB-7 } & -152.03 & 71.05 & 1.00 & 0.11 \\ \text { Ik } & -151.08 & 72.02 & & \end{array}$

Glacial-interglacial contrast in MBT/CBT proxies in the South China Sea: Implications for marine producti

\begin{tabular}{|c|c|c|c|c|}
\hline 0 & 8.82 & 111.43 & 2.28 & 0.08 \\
\hline 2 & 8.82 & 111.43 & & \\
\hline 4 & 8.82 & 111.43 & 3.30 & 0.06 \\
\hline 6 & 8.82 & 111.43 & 2.42 & 0.04 \\
\hline 8 & 8.82 & 111.43 & 2.99 & 0.04 \\
\hline 10 & 8.82 & 111.43 & 2.88 & 0.04 \\
\hline 12 & 8.82 & 111.43 & 3.32 & 0.04 \\
\hline 14 & 8.82 & 111.43 & 3.58 & 0.05 \\
\hline 16 & 8.82 & 111.43 & 3.27 & 0.04 \\
\hline 18 & 8.82 & 111.43 & 2.94 & 0.04 \\
\hline 20 & 8.82 & 111.43 & 3.76 & 0.05 \\
\hline 22 & 8.82 & 111.43 & & \\
\hline 24 & 8.82 & 111.43 & 3.00 & 0.05 \\
\hline 26 & 8.82 & 111.43 & & \\
\hline 28 & 8.82 & 111.43 & 2.96 & 0.05 \\
\hline 30 & 8.82 & 111.43 & 2.69 & 0.05 \\
\hline 32 & 8.82 & 111.43 & & \\
\hline 34 & 8.82 & 111.43 & 2.31 & 0.05 \\
\hline 36 & 8.82 & 111.43 & & \\
\hline 38 & 8.82 & 111.43 & & \\
\hline 40 & 8.82 & 111.43 & 2.97 & 0.05 \\
\hline 42 & 8.82 & 111.43 & 2.81 & 0.05 \\
\hline 44 & 8.82 & 111.43 & 2.78 & 0.05 \\
\hline 46 & 8.82 & 111.43 & 3.19 & 0.06 \\
\hline 48 & 8.82 & 111.43 & & \\
\hline 50 & 8.82 & 111.43 & 3.38 & 0.05 \\
\hline 52 & 8.82 & 111.43 & 3.05 & 0.06 \\
\hline 54 & 8.82 & 111.43 & 2.80 & 0.03 \\
\hline 56 & 8.82 & 111.43 & 2.80 & 0.0 \\
\hline
\end{tabular}




\begin{tabular}{|c|c|c|c|c|}
\hline 58 & 8.82 & 111.43 & 3.06 & 0.05 \\
\hline 60 & 8.82 & 111.43 & 3.19 & 0.05 \\
\hline 62 & 8.82 & 111.43 & 3.21 & 0.04 \\
\hline 64 & 8.82 & 111.43 & 2.84 & 0.04 \\
\hline 66 & 8.82 & 111.43 & 2.76 & 0.05 \\
\hline 68 & 8.82 & 111.43 & 2.53 & 0.05 \\
\hline 70 & 8.82 & 111.43 & 2.91 & 0.07 \\
\hline 72 & 8.82 & 111.43 & 2.90 & 0.06 \\
\hline 74 & 8.82 & 111.43 & 3.01 & 0.06 \\
\hline 76 & 8.82 & 111.43 & 3.28 & 0.05 \\
\hline 78 & 8.82 & 111.43 & 3.02 & 0.06 \\
\hline 80 & 8.82 & 111.43 & 3.11 & 0.07 \\
\hline 82 & 8.82 & 111.43 & 2.87 & 0.05 \\
\hline 84 & 8.82 & 111.43 & 2.88 & 0.07 \\
\hline 86 & 8.82 & 111.43 & 3.04 & 0.07 \\
\hline 88 & 8.82 & 111.43 & 3.12 & 0.07 \\
\hline 90 & 8.82 & 111.43 & 3.02 & 0.06 \\
\hline 92 & 8.82 & 111.43 & 3.07 & 0.07 \\
\hline 94 & 8.82 & 111.43 & 2.81 & 0.06 \\
\hline 96 & 8.82 & 111.43 & 2.97 & 0.05 \\
\hline 98 & 8.82 & 111.43 & 2.75 & 0.06 \\
\hline 100 & 8.82 & 111.43 & & \\
\hline 102 & 8.82 & 111.43 & 2.12 & 0.09 \\
\hline 104 & 8.82 & 111.43 & 2.17 & 0.07 \\
\hline 106 & 8.82 & 111.43 & 2.26 & 0.08 \\
\hline 108 & 8.82 & 111.43 & 2.17 & 0.08 \\
\hline 110 & 8.82 & 111.43 & & \\
\hline 112 & 8.82 & 111.43 & & \\
\hline 114 & 8.82 & 111.43 & 1.98 & 0.08 \\
\hline 116 & 8.82 & 111.43 & 2.06 & 0.12 \\
\hline 118 & 8.82 & 111.43 & & 0.10 \\
\hline 120 & 8.82 & 111.43 & 2.21 & 0.12 \\
\hline 122 & 8.82 & 111.43 & 1.83 & 0.10 \\
\hline 124 & 8.82 & 111.43 & 1.66 & 0.13 \\
\hline 126 & 8.82 & 111.43 & 1.90 & 0.36 \\
\hline 128 & 8.82 & 111.43 & 1.75 & 0.11 \\
\hline 130 & 8.82 & 111.43 & 1.95 & 0.11 \\
\hline 132 & 8.82 & 111.43 & 1.78 & 0.12 \\
\hline 134 & 8.82 & 111.43 & 1.82 & 0.11 \\
\hline 136 & 8.82 & 111.43 & 1.56 & 0.11 \\
\hline 138 & 8.82 & 111.43 & 1.48 & 0.12 \\
\hline 140 & 8.82 & 111.43 & 1.71 & 0.10 \\
\hline 142 & 8.82 & 111.43 & 1.60 & 0.13 \\
\hline 144 & 8.82 & 111.43 & \#DIV/0! & \\
\hline 146 & 8.82 & 111.43 & 1.57 & 0.11 \\
\hline 148 & 8.82 & 111.43 & \#DIV/0! & \\
\hline 150 & 8.82 & 111.43 & 1.38 & 0.12 \\
\hline 152 & 8.82 & 111.43 & 1.25 & 0.14 \\
\hline 154 & 8.82 & 111.43 & 1.69 & 0.09 \\
\hline 156 & 8.82 & 111.43 & 1.65 & 0.10 \\
\hline 158 & 8.82 & 111.43 & 1.71 & 0.10 \\
\hline 160 & 8.82 & 111.43 & 1.50 & 0.11 \\
\hline 162 & 8.82 & 111.43 & 1.52 & 0.12 \\
\hline 164 & 8.82 & 111.43 & 1.84 & 0.11 \\
\hline
\end{tabular}




$\begin{array}{lllll}166 & 8.82 & 111.43 & 1.63 & 0.14 \\ 168 & 8.82 & 111.43 & 1.67 & 0.14 \\ 170 & 8.82 & 111.43 & 1.67 & 0.15 \\ 172 & 8.82 & 111.43 & 1.58 & 0.17 \\ 174 & 8.82 & 111.43 & 1.56 & 0.12 \\ 176 & 8.82 & 111.43 & 1.42 & 0.13 \\ 178 & 8.82 & 111.43 & 1.51 & 0.13 \\ 180 & 8.82 & 111.43 & 1.32 & 0.13 \\ 182 & 8.82 & 111.43 & 1.35 & 0.12 \\ 184 & 8.82 & 111.43 & 1.67 & 0.12 \\ 186 & 8.82 & 111.43 & 1.63 & 0.12 \\ 188 & 8.82 & 111.43 & 1.18 & 0.12 \\ 190 & 8.82 & 111.43 & 1.61 & 0.12 \\ 192 & 8.82 & 111.43 & 1.65 & 0.13 \\ 194 & 8.82 & 111.43 & 1.24 & 0.15 \\ 196 & 8.82 & 111.43 & 1.54 & 0.14 \\ 198 & 8.82 & 111.43 & 1.73 & 0.14 \\ 70 & 8.82 & 111.43 & 0.05 & 0.18 \\ 72 & 8.82 & 111.43 & 1.23 & 0.14 \\ 74 & 8.82 & 111.43 & 1.09 & 0.22 \\ 76 & 8.82 & 111.43 & 1.47 & 0.12 \\ 78 & 8.82 & 111.43 & 0.05 & 0.11 \\ 80 & 8.82 & 111.43 & 1.38 & 0.13 \\ 82 & 8.82 & 111.43 & 1.72 & 0.15 \\ 84 & 8.82 & 111.43 & 5.05 & 0.14 \\ 86 & 8.82 & 111.43 & 1.45 & 0.12 \\ 88 & 8.82 & 111.43 & 1.48 & 0.10 \\ 90 & 8.82 & 111.43 & 1.08 & 0.17 \\ 92 & 8.82 & 111.43 & 1.30 & 0.14 \\ 94 & 8.82 & 111.43 & 1.19 & 0.19 \\ 96 & 8.82 & 111.43 & 1.02 & 0.28 \\ 98 & 8.82 & 111.43 & 1.31 & 0.15 \\ 100 & 8.82 & 111.43 & 1.09 & 0.24 \\ 102 & 8.82 & 111.43 & 1.52 & 0.12 \\ 104 & 8.82 & 111.43 & 1.40 & 0.17 \\ 106 & 8.82 & 111.43 & 1.70 & 0.11 \\ 108 & 8.82 & 111.43 & 1.45 & 0.11 \\ 110 & 8.82 & 111.43 & & \\ 112 & 8.82 & 111.43 & & \\ 114 & 8.82 & 111.43 & & \\ 116 & 8.82 & 111.43 & 1.11 & 0.20 \\ 118 & 8.82 & 111.43 & 0.94 & 0.34 \\ 120 & 8.82 & 111.43 & 1.18 & 0.21 \\ 122 & 8.82 & 111.43 & & \\ 124 & 8.82 & 111.43 & 0.89 & 0.29 \\ 126 & 8.82 & 111.43 & 1.21 & 0.16 \\ 128 & 8.82 & 111.43 & 1.25 & 0.14 \\ 130 & 8.82 & 111.43 & 0.92 & 0.26 \\ 132 & 8.82 & 111.43 & 1.29 & 0.12 \\ 134 & 8.82 & 111.43 & 1.43 & 0.11 \\ 136 & 8.82 & 111.43 & 0.87 & 0.27 \\ 138 & 8.82 & 111.43 & 1.13 & 0.12 \\ 142 & 8.82 & 111.43 & 1.12 & 0.18 \\ & & 111.43 & 1.28 & 0.10\end{array}$




\begin{tabular}{|c|c|c|c|c|}
\hline 144 & 8.82 & 111.43 & 1.40 & 0.11 \\
\hline 146 & 8.82 & 111.43 & 1.03 & 0.25 \\
\hline 148 & 8.82 & 111.43 & 1.09 & 0.21 \\
\hline 150 & 8.82 & 111.43 & 0.92 & 0.19 \\
\hline 152 & 8.82 & 111.43 & 0.95 & 0.19 \\
\hline 154 & 8.82 & 111.43 & 1.36 & 0.13 \\
\hline 156 & 8.82 & 111.43 & 1.22 & 0.17 \\
\hline 158 & 8.82 & 111.43 & 1.17 & 0.23 \\
\hline 160 & 8.82 & 111.43 & 1.34 & 0.24 \\
\hline 162 & 8.82 & 111.43 & 1.59 & 0.15 \\
\hline 164 & 8.82 & 111.43 & & \\
\hline 166 & 8.82 & 111.43 & 1.60 & 0.20 \\
\hline 168 & 8.82 & 111.43 & 1.13 & 0.15 \\
\hline 170 & 8.82 & 111.43 & 1.34 & 0.09 \\
\hline 172 & 8.82 & 111.43 & 1.23 & 0.12 \\
\hline 174 & 8.82 & 111.43 & 1.22 & 0.13 \\
\hline 176 & 8.82 & 111.43 & 1.74 & 0.07 \\
\hline 178 & 8.82 & 111.43 & 1.32 & 0.11 \\
\hline 180 & 8.82 & 111.43 & 1.68 & 0.08 \\
\hline 182 & 8.82 & 111.43 & 1.40 & 0.09 \\
\hline 184 & 8.82 & 111.43 & 1.11 & 0.15 \\
\hline 186 & 8.82 & 111.43 & 1.30 & 0.14 \\
\hline 188 & 8.82 & 111.43 & 1.50 & 0.14 \\
\hline 190 & 8.82 & 111.43 & 1.17 & 0.15 \\
\hline 192 & 8.82 & 111.43 & 1.50 & 0.14 \\
\hline 194 & 8.82 & 111.43 & & \\
\hline 196 & 8.82 & 111.43 & 1.28 & 0.09 \\
\hline 198 & 8.82 & 111.43 & 1.44 & 0.09 \\
\hline 200 & 8.82 & 111.43 & 1.13 & 0.16 \\
\hline 202 & 8.82 & 111.43 & 1.48 & 0.09 \\
\hline 204 & 8.82 & 111.43 & 1.23 & 0.11 \\
\hline 206 & 8.82 & 111.43 & 1.29 & 0.13 \\
\hline 208 & 8.82 & 111.43 & 1.43 & 0.09 \\
\hline 210 & 8.82 & 111.43 & 1.22 & 0.08 \\
\hline 212 & 8.82 & 111.43 & 1.09 & 0.12 \\
\hline 214 & 8.82 & 111.43 & 0.97 & 0.15 \\
\hline 216 & 8.82 & 111.43 & 1.20 & 0.10 \\
\hline 218 & 8.82 & 111.43 & 1.58 & 0.08 \\
\hline 220 & 8.82 & 111.43 & 0.69 & 0.12 \\
\hline 222 & 8.82 & 111.43 & 0.91 & 0.13 \\
\hline 224 & 8.82 & 111.43 & 1.29 & 0.08 \\
\hline 226 & 8.82 & 111.43 & 0.85 & 0.16 \\
\hline 228 & 8.82 & 111.43 & & \\
\hline 230 & 8.82 & 111.43 & 0.85 & 0.15 \\
\hline 232 & 8.82 & 111.43 & 0.77 & 0.16 \\
\hline 234 & 8.82 & 111.43 & 0.72 & 0.06 \\
\hline 236 & 8.82 & 111.43 & 0.89 & 0.16 \\
\hline 238 & 8.82 & 111.43 & 1.21 & 0.10 \\
\hline 240 & 8.82 & 111.43 & 0.94 & 0.16 \\
\hline 242 & 8.82 & 111.43 & 0.91 & 0.18 \\
\hline 244 & 8.82 & 111.43 & 1.23 & 0.06 \\
\hline 246 & 8.82 & 111.43 & 1.18 & 0.11 \\
\hline 248 & 8.82 & 111.43 & 1.24 & 0.15 \\
\hline 250 & 8.82 & 111.43 & 1.40 & 0.09 \\
\hline
\end{tabular}




\begin{tabular}{|c|c|c|c|c|}
\hline 252 & 8.82 & 111.43 & 1.38 & 0.11 \\
\hline 254 & 8.82 & 111.43 & 1.06 & 0.15 \\
\hline 256 & 8.82 & 111.43 & & \\
\hline 258 & 8.82 & 111.43 & 1.48 & 0.08 \\
\hline 260 & 8.82 & 111.43 & 1.08 & 0.15 \\
\hline 262 & 8.82 & 111.43 & 0.97 & 0.15 \\
\hline 264 & 8.82 & 111.43 & 1.10 & 0.17 \\
\hline 266 & 8.82 & 111.43 & 1.16 & 0.11 \\
\hline 268 & 8.82 & 111.43 & 1.21 & 0.06 \\
\hline 270 & 8.82 & 111.43 & 0.99 & 0.07 \\
\hline 272 & 8.82 & 111.43 & 1.38 & 0.15 \\
\hline 274 & 8.82 & 111.43 & 1.19 & 0.10 \\
\hline 276 & 8.82 & 111.43 & 1.50 & 0.05 \\
\hline 278 & 8.82 & 111.43 & 1.49 & 0.06 \\
\hline 280 & 8.82 & 111.43 & 1.25 & 0.09 \\
\hline 282 & 8.82 & 111.43 & 1.27 & 0.10 \\
\hline 284 & 8.82 & 111.43 & 1.63 & 0.06 \\
\hline 286 & 8.82 & 111.43 & 1.40 & 0.08 \\
\hline 288 & 8.82 & 111.43 & 1.90 & 0.07 \\
\hline 290 & 8.82 & 111.43 & 1.45 & 0.09 \\
\hline 292 & 8.82 & 111.43 & 1.56 & 0.06 \\
\hline 294 & 8.82 & 111.43 & 1.54 & 0.05 \\
\hline 296 & 8.82 & 111.43 & 1.09 & 0.11 \\
\hline 298 & 8.82 & 111.43 & 1.28 & 0.16 \\
\hline 300 & 8.82 & 111.43 & 1.26 & 0.09 \\
\hline 302 & 8.82 & 111.43 & & \\
\hline 304 & 8.82 & 111.43 & 1.42 & 0.07 \\
\hline 306 & 8.82 & 111.43 & 1.30 & 0.07 \\
\hline 308 & 8.82 & 111.43 & 1.03 & 0.12 \\
\hline 310 & 8.82 & 111.43 & 1.34 & 0.09 \\
\hline 312 & 8.82 & 111.43 & 1.27 & 0.10 \\
\hline 314 & 8.82 & 111.43 & 1.34 & 0.07 \\
\hline 316 & 8.82 & 111.43 & 0.96 & 0.09 \\
\hline 318 & 8.82 & 111.43 & 1.23 & 0.09 \\
\hline 320 & 8.82 & 111.43 & 0.94 & 0.15 \\
\hline 322 & 8.82 & 111.43 & 1.40 & 0.10 \\
\hline 324 & 8.82 & 111.43 & 1.04 & 0.13 \\
\hline 326 & 8.82 & 111.43 & 1.18 & 0.10 \\
\hline 328 & 8.82 & 111.43 & 1.13 & 0.12 \\
\hline 330 & 8.82 & 111.43 & 1.17 & 0.18 \\
\hline 332 & 8.82 & 111.43 & 1.07 & 0.20 \\
\hline 334 & 8.82 & 111.43 & 1.51 & 0.08 \\
\hline 336 & 8.82 & 111.43 & 1.68 & 0.10 \\
\hline 338 & 8.82 & 111.43 & 0.96 & 0.10 \\
\hline 340 & 8.82 & 111.43 & 1.70 & 0.07 \\
\hline 342 & 8.82 & 111.43 & 1.16 & 0.15 \\
\hline 344 & 8.82 & 111.43 & 1.07 & 0.11 \\
\hline 346 & 8.82 & 111.43 & 1.46 & 0.09 \\
\hline 348 & 8.82 & 111.43 & 1.30 & 0.13 \\
\hline 350 & 8.82 & 111.43 & 1.19 & 0.15 \\
\hline 352 & 8.82 & 111.43 & 1.81 & 0.10 \\
\hline 354 & 8.82 & 111.43 & 1.41 & 0.10 \\
\hline 356 & 8.82 & 111.43 & 1.02 & 0.09 \\
\hline 358 & 8.82 & 111.43 & 1.46 & 0.05 \\
\hline
\end{tabular}




\begin{tabular}{|c|c|c|c|c|}
\hline 360 & 8.82 & 111.43 & 1.22 & 0.09 \\
\hline 362 & 8.82 & 111.43 & 1.21 & 0.09 \\
\hline 364 & 8.82 & 111.43 & 1.94 & 0.08 \\
\hline 366 & 8.82 & 111.43 & 2.07 & 0.06 \\
\hline 368 & 8.82 & 111.43 & 1.71 & 0.05 \\
\hline 370 & 8.82 & 111.43 & 1.77 & 0.05 \\
\hline 372 & 8.82 & 111.43 & 1.25 & 0.11 \\
\hline 374 & 8.82 & 111.43 & 1.15 & 0.08 \\
\hline 376 & 8.82 & 111.43 & 1.27 & 0.10 \\
\hline 378 & 8.82 & 111.43 & 2.01 & 0.07 \\
\hline 380 & 8.82 & 111.43 & 1.99 & 0.06 \\
\hline 382 & 8.82 & 111.43 & 1.47 & 0.12 \\
\hline 384 & 8.82 & 111.43 & 1.80 & 0.06 \\
\hline 386 & 8.82 & 111.43 & 1.01 & 0.15 \\
\hline 388 & 8.82 & 111.43 & 1.17 & 0.09 \\
\hline 390 & 8.82 & 111.43 & 1.23 & 0.10 \\
\hline 392 & 8.82 & 111.43 & 1.24 & 0.12 \\
\hline 394 & 8.82 & 111.43 & 1.13 & 0.12 \\
\hline 396 & 8.82 & 111.43 & 1.07 & 0.13 \\
\hline 398 & 8.82 & 111.43 & 1.14 & 0.11 \\
\hline 400 & 8.82 & 111.43 & 1.52 & 0.07 \\
\hline 402 & 8.82 & 111.43 & 1.32 & 0.10 \\
\hline 404 & 8.82 & 111.43 & 1.35 & 0.10 \\
\hline 406 & 8.82 & 111.43 & 1.56 & 0.07 \\
\hline 408 & 8.82 & 111.43 & 1.69 & 0.06 \\
\hline 410 & 8.82 & 111.43 & 1.16 & 0.10 \\
\hline 412 & 8.82 & 111.43 & 1.34 & 0.11 \\
\hline 414 & 8.82 & 111.43 & 1.81 & 0.08 \\
\hline 416 & 8.82 & 111.43 & 1.00 & 0.14 \\
\hline 418 & 8.82 & 111.43 & 1.32 & 0.09 \\
\hline 420 & 8.82 & 111.43 & 1.29 & 0.06 \\
\hline 422 & 8.82 & 111.43 & 1.67 & 0.06 \\
\hline 424 & 8.82 & 111.43 & 1.52 & 0.07 \\
\hline 426 & 8.82 & 111.43 & 1.34 & 0.08 \\
\hline 428 & 8.82 & 111.43 & 1.48 & 0.06 \\
\hline 430 & 8.82 & 111.43 & 1.17 & 0.11 \\
\hline 432 & 8.82 & 111.43 & 1.48 & 0.07 \\
\hline 434 & 8.82 & 111.43 & 1.58 & 0.06 \\
\hline 436 & 8.82 & 111.43 & 1.40 & 0.09 \\
\hline 438 & 8.82 & 111.43 & 1.39 & 0.06 \\
\hline 440 & 8.82 & 111.43 & 1.16 & 0.09 \\
\hline 442 & 8.82 & 111.43 & 1.70 & 0.06 \\
\hline 444 & 8.82 & 111.43 & 1.41 & 0.05 \\
\hline 446 & 8.82 & 111.43 & 1.22 & 0.07 \\
\hline 448 & 8.82 & 111.43 & 1.08 & 0.07 \\
\hline 450 & 8.82 & 111.43 & 1.37 & 0.07 \\
\hline 452 & 8.82 & 111.43 & 1.52 & 0.08 \\
\hline 454 & 8.82 & 111.43 & 1.34 & 0.08 \\
\hline 456 & 8.82 & 111.43 & 1.33 & 0.07 \\
\hline 458 & 8.82 & 111.43 & 1.49 & 0.09 \\
\hline 460 & 8.82 & 111.43 & 1.49 & 0.09 \\
\hline 462 & 8.82 & 111.43 & 1.30 & 0.08 \\
\hline 464 & 8.82 & 111.43 & 1.31 & 0.13 \\
\hline 466 & 8.82 & 111.43 & 1.16 & 0.11 \\
\hline
\end{tabular}




\begin{tabular}{|c|c|c|c|c|}
\hline 468 & 8.82 & 111.43 & 1.26 & 0.09 \\
\hline 470 & 8.82 & 111.43 & 1.33 & 0.08 \\
\hline 472 & 8.82 & 111.43 & 1.36 & 0.11 \\
\hline 474 & 8.82 & 111.43 & 1.35 & 0.07 \\
\hline 476 & 8.82 & 111.43 & 1.30 & 0.08 \\
\hline 478 & 8.82 & 111.43 & 1.37 & 0.07 \\
\hline 480 & 8.82 & 111.43 & 1.28 & 0.08 \\
\hline 482 & 8.82 & 111.43 & 1.14 & 0.08 \\
\hline 484 & 8.82 & 111.43 & 1.18 & 0.08 \\
\hline 486 & 8.82 & 111.43 & 1.20 & 0.09 \\
\hline 488 & 8.82 & 111.43 & 1.10 & 0.11 \\
\hline 490 & 8.82 & 111.43 & 1.23 & 0.12 \\
\hline 492 & 8.82 & 111.43 & 1.10 & 0.15 \\
\hline 494 & 8.82 & 111.43 & 1.23 & 0.13 \\
\hline 496 & 8.82 & 111.43 & 1.34 & 0.14 \\
\hline 498 & 8.82 & 111.43 & 1.32 & 0.12 \\
\hline 500 & 8.82 & 111.43 & 1.23 & 0.11 \\
\hline 502 & 8.82 & 111.43 & 1.22 & 0.12 \\
\hline 504 & 8.82 & 111.43 & 1.29 & 0.10 \\
\hline 506 & 8.82 & 111.43 & 1.19 & 0.12 \\
\hline 508 & 8.82 & 111.43 & 1.17 & 0.09 \\
\hline 510 & 8.82 & 111.43 & 1.20 & 0.09 \\
\hline 512 & 8.82 & 111.43 & 1.14 & 0.09 \\
\hline 514 & 8.82 & 111.43 & 1.10 & 0.14 \\
\hline 516 & 8.82 & 111.43 & 1.05 & 0.08 \\
\hline 518 & 8.82 & 111.43 & 1.07 & 0.12 \\
\hline 520 & 8.82 & 111.43 & 1.42 & 0.09 \\
\hline 522 & 8.82 & 111.43 & 1.02 & 0.13 \\
\hline 524 & 8.82 & 111.43 & 1.21 & 0.09 \\
\hline 526 & 8.82 & 111.43 & 1.27 & 0.10 \\
\hline 528 & 8.82 & 111.43 & 1.02 & 0.11 \\
\hline 530 & 8.82 & 111.43 & 1.10 & 0.09 \\
\hline 532 & 8.82 & 111.43 & 1.25 & 0.09 \\
\hline 534 & 8.82 & 111.43 & 1.22 & 0.10 \\
\hline 536 & 8.82 & 111.43 & 1.16 & 0.10 \\
\hline 538 & 8.82 & 111.43 & & \\
\hline 540 & 8.82 & 111.43 & 0.89 & 0.16 \\
\hline 542 & 8.82 & 111.43 & 1.17 & 0.11 \\
\hline 544 & 8.82 & 111.43 & 1.15 & 0.11 \\
\hline 546 & 8.82 & 111.43 & 0.97 & 0.12 \\
\hline 548 & 8.82 & 111.43 & 0.87 & 0.10 \\
\hline 550 & 8.82 & 111.43 & 0.94 & 0.16 \\
\hline 552 & 8.82 & 111.43 & 1.14 & 0.13 \\
\hline 554 & 8.82 & 111.43 & 1.25 & 0.11 \\
\hline 556 & 8.82 & 111.43 & 1.17 & 0.11 \\
\hline 558 & 8.82 & 111.43 & 1.09 & 0.11 \\
\hline 560 & 8.82 & 111.43 & 1.31 & 0.09 \\
\hline 562 & 8.82 & 111.43 & 1.23 & 0.10 \\
\hline 564 & 8.82 & 111.43 & 1.25 & 0.09 \\
\hline 566 & 8.82 & 111.43 & 1.21 & 0.09 \\
\hline 568 & 8.82 & 111.43 & 1.18 & 0.09 \\
\hline 570 & 8.82 & 111.43 & 1.21 & 0.06 \\
\hline 572 & 8.82 & 111.43 & 1.04 & 0.06 \\
\hline 574 & 8.82 & 111.43 & 1.02 & 0.06 \\
\hline
\end{tabular}




\begin{tabular}{|c|c|c|c|c|}
\hline 576 & 8.82 & 111.43 & 0.99 & 0.07 \\
\hline 578 & 8.82 & 111.43 & 1.19 & 0.08 \\
\hline 580 & 8.82 & 111.43 & \#DIV/0! & 0.08 \\
\hline 582 & 8.82 & 111.43 & 1.49 & 0.06 \\
\hline 584 & 8.82 & 111.43 & 1.56 & 0.06 \\
\hline 586 & 8.82 & 111.43 & 1.35 & 0.08 \\
\hline 588 & 8.82 & 111.43 & 1.57 & 0.06 \\
\hline 590 & 8.82 & 111.43 & 1.57 & 0.07 \\
\hline 592 & 8.82 & 111.43 & 1.61 & 0.05 \\
\hline 594 & 8.82 & 111.43 & 1.46 & 0.05 \\
\hline 596 & 8.82 & 111.43 & 1.62 & 0.05 \\
\hline 598 & 8.82 & 111.43 & 1.49 & 0.07 \\
\hline 600 & 8.82 & 111.43 & & \\
\hline 602 & 8.82 & 111.43 & 1.40 & 0.06 \\
\hline 604 & 8.82 & 111.43 & 1.25 & 0.06 \\
\hline 606 & 8.82 & 111.43 & & \\
\hline 608 & 8.82 & 111.43 & 2.08 & 0.04 \\
\hline 610 & 8.82 & 111.43 & & \\
\hline 612 & 8.82 & 111.43 & 1.64 & 0.04 \\
\hline 614 & 8.82 & 111.43 & 1.12 & 0.05 \\
\hline 616 & 8.82 & 111.43 & 1.50 & 0.05 \\
\hline 618 & 8.82 & 111.43 & 1.94 & 0.04 \\
\hline 620 & 8.82 & 111.43 & & \\
\hline 622 & 8.82 & 111.43 & 1.61 & 0.04 \\
\hline 624 & 8.82 & 111.43 & 1.69 & 0.05 \\
\hline 626 & 8.82 & 111.43 & 1.55 & 0.05 \\
\hline 628 & 8.82 & 111.43 & & \\
\hline 630 & 8.82 & 111.43 & 1.82 & 0.04 \\
\hline 632 & 8.82 & 111.43 & 1.80 & 0.04 \\
\hline 634 & 8.82 & 111.43 & 1.89 & 0.04 \\
\hline 636 & 8.82 & 111.43 & 1.94 & 0.03 \\
\hline 638 & 8.82 & 111.43 & 2.04 & 0.04 \\
\hline 640 & 8.82 & 111.43 & 2.18 & 0.04 \\
\hline 642 & 8.82 & 111.43 & 2.19 & 0.05 \\
\hline 644 & 8.82 & 111.43 & 1.81 & 0.04 \\
\hline 646 & 8.82 & 111.43 & & 0.04 \\
\hline 648 & 8.82 & 111.43 & 1.98 & 0.05 \\
\hline 650 & 8.82 & 111.43 & 2.00 & 0.05 \\
\hline 652 & 8.82 & 111.43 & 1.64 & 0.05 \\
\hline 654 & 8.82 & 111.43 & 2.09 & 0.05 \\
\hline 656 & 8.82 & 111.43 & 1.95 & 0.04 \\
\hline 658 & 8.82 & 111.43 & 1.94 & 0.03 \\
\hline 660 & 8.82 & 111.43 & 2.04 & 0.04 \\
\hline 662 & 8.82 & 111.43 & 2.12 & 0.04 \\
\hline 664 & 8.82 & 111.43 & 2.14 & 0.05 \\
\hline 666 & 8.82 & 111.43 & 2.32 & 0.05 \\
\hline 668 & 8.82 & 111.43 & 2.21 & 0.03 \\
\hline 670 & 8.82 & 111.43 & 2.14 & 0.04 \\
\hline 672 & 8.82 & 111.43 & 2.27 & 0.04 \\
\hline 674 & 8.82 & 111.43 & 2.55 & 0.04 \\
\hline 676 & 8.82 & 111.43 & 2.60 & 0.04 \\
\hline 678 & 8.82 & 111.43 & 2.61 & 0.05 \\
\hline 680 & 8.82 & 111.43 & 2.35 & 0.04 \\
\hline 682 & 8.82 & 111.43 & 2.41 & 0.05 \\
\hline
\end{tabular}




\begin{tabular}{|c|c|c|c|c|}
\hline 684 & 8.82 & 111.43 & 2.23 & 0.04 \\
\hline 686 & 8.82 & 111.43 & 2.15 & 0.04 \\
\hline 688 & 8.82 & 111.43 & 1.40 & 0.04 \\
\hline 690 & 8.82 & 111.43 & 2.21 & 0.03 \\
\hline 692 & 8.82 & 111.43 & 2.46 & 0.04 \\
\hline 694 & 8.82 & 111.43 & & \\
\hline 696 & 8.82 & 111.43 & 1.24 & 0.05 \\
\hline 698 & 8.82 & 111.43 & 2.67 & 0.05 \\
\hline 700 & 8.82 & 111.43 & 2.52 & 0.05 \\
\hline 702 & 8.82 & 111.43 & 2.53 & 0.05 \\
\hline 704 & 8.82 & 111.43 & 2.34 & 0.05 \\
\hline 706 & 8.82 & 111.43 & 2.57 & 0.05 \\
\hline 708 & 8.82 & 111.43 & 2.57 & 0.06 \\
\hline 710 & 8.82 & 111.43 & 2.24 & 0.06 \\
\hline 712 & 8.82 & 111.43 & 2.55 & 0.06 \\
\hline 714 & 8.82 & 111.43 & 2.38 & 0.06 \\
\hline 716 & 8.82 & 111.43 & 2.58 & 0.07 \\
\hline 718 & 8.82 & 111.43 & 2.04 & 0.06 \\
\hline 720 & 8.82 & 111.43 & 1.97 & 0.06 \\
\hline 722 & 8.82 & 111.43 & 2.28 & 0.05 \\
\hline 724 & 8.82 & 111.43 & 2.16 & 0.06 \\
\hline 726 & 8.82 & 111.43 & 1.96 & 0.07 \\
\hline 728 & 8.82 & 111.43 & 2.12 & 0.05 \\
\hline 730 & 8.82 & 111.43 & 2.22 & 0.05 \\
\hline 732 & 8.82 & 111.43 & 2.09 & 0.05 \\
\hline 734 & 8.82 & 111.43 & 1.94 & 0.05 \\
\hline 736 & 8.82 & 111.43 & & \\
\hline 738 & 8.82 & 111.43 & & \\
\hline 740 & 8.82 & 111.43 & 2.06 & 0.05 \\
\hline 742 & 8.82 & 111.43 & & \\
\hline 744 & 8.82 & 111.43 & 1.95 & 0.04 \\
\hline 746 & 8.82 & 111.43 & 2.05 & 0.04 \\
\hline 748 & 8.82 & 111.43 & 2.18 & 0.04 \\
\hline 750 & 8.82 & 111.43 & 2.16 & 0.05 \\
\hline 752 & 8.82 & 111.43 & 2.09 & 0.04 \\
\hline 754 & 8.82 & 111.43 & 2.13 & 0.04 \\
\hline 756 & 8.82 & 111.43 & 2.02 & 0.05 \\
\hline 758 & 8.82 & 111.43 & 2.19 & 0.05 \\
\hline 760 & 8.82 & 111.43 & 2.22 & 0.05 \\
\hline 762 & 8.82 & 111.43 & 2.23 & 0.06 \\
\hline 764 & 8.82 & 111.43 & 2.21 & 0.05 \\
\hline 766 & 8.82 & 111.43 & 2.15 & 0.06 \\
\hline 768 & 8.82 & 111.43 & 2.08 & 0.04 \\
\hline 770 & 8.82 & 111.43 & 1.93 & 0.03 \\
\hline 772 & 8.82 & 111.43 & & \\
\hline 774 & 8.82 & 111.43 & 2.19 & 0.04 \\
\hline 776 & 8.82 & 111.43 & 2.07 & 0.04 \\
\hline 778 & 8.82 & 111.43 & 2.19 & 0.04 \\
\hline 780 & 8.82 & 111.43 & 2.12 & 0.04 \\
\hline 782 & 8.82 & 111.43 & 1.99 & 0.04 \\
\hline 784 & 8.82 & 111.43 & 2.32 & 0.04 \\
\hline 786 & 8.82 & 111.43 & 2.10 & 0.05 \\
\hline 788 & 8.82 & 111.43 & 2.21 & 0.05 \\
\hline 790 & 8.82 & 111.43 & 1.91 & 0.06 \\
\hline
\end{tabular}




\begin{tabular}{|c|c|c|c|c|}
\hline 792 & 8.82 & 111.43 & 2.13 & 0.05 \\
\hline 794 & 8.82 & 111.43 & 1.77 & 0.03 \\
\hline 796 & 8.82 & 111.43 & 1.99 & 0.04 \\
\hline 798 & 8.82 & 111.43 & 2.18 & 0.04 \\
\hline 800 & 8.82 & 111.43 & 2.15 & 0.04 \\
\hline 802 & 8.82 & 111.43 & 2.19 & 0.05 \\
\hline 804 & 8.82 & 111.43 & 2.29 & 0.05 \\
\hline 806 & 8.82 & 111.43 & 2.16 & 0.04 \\
\hline 808 & 8.82 & 111.43 & 2.48 & 0.04 \\
\hline 810 & 8.82 & 111.43 & 1.19 & 0.03 \\
\hline 812 & 8.82 & 111.43 & 2.30 & 0.03 \\
\hline 814 & 8.82 & 111.43 & 2.29 & 0.04 \\
\hline 816 & 8.82 & 111.43 & 2.31 & 0.04 \\
\hline 818 & 8.82 & 111.43 & 2.21 & 0.05 \\
\hline 820 & 8.82 & 111.43 & 2.61 & 0.04 \\
\hline 822 & 8.82 & 111.43 & 2.53 & 0.04 \\
\hline 824 & 8.82 & 111.43 & 2.38 & 0.05 \\
\hline 826 & 8.82 & 111.43 & 2.40 & 0.05 \\
\hline 828 & 8.82 & 111.43 & 2.47 & 0.06 \\
\hline 830 & 8.82 & 111.43 & 2.36 & 0.05 \\
\hline 832 & 8.82 & 111.43 & 2.64 & 0.05 \\
\hline 834 & 8.82 & 111.43 & 2.38 & 0.05 \\
\hline 836 & 8.82 & 111.43 & 2.62 & 0.05 \\
\hline 838 & 8.82 & 111.43 & 2.51 & 0.05 \\
\hline 840 & 8.82 & 111.43 & 2.30 & 0.04 \\
\hline 842 & 8.82 & 111.43 & 2.38 & 0.04 \\
\hline 844 & 8.82 & 111.43 & 2.11 & 0.04 \\
\hline 846 & 8.82 & 111.43 & 2.40 & 0.05 \\
\hline 848 & 8.82 & 111.43 & 2.26 & 0.04 \\
\hline 850 & 8.82 & 111.43 & 2.10 & 0.05 \\
\hline 852 & 8.82 & 111.43 & 2.47 & 0.05 \\
\hline 854 & 8.82 & 111.43 & 2.26 & 0.05 \\
\hline 856 & 8.82 & 111.43 & 2.39 & 0.05 \\
\hline 858 & 8.82 & 111.43 & 2.56 & 0.06 \\
\hline 860 & 8.82 & 111.43 & 2.24 & 0.07 \\
\hline 862 & 8.82 & 111.43 & 2.54 & 0.07 \\
\hline 864 & 8.82 & 111.43 & 2.59 & 0.06 \\
\hline 866 & 8.82 & 111.43 & 2.42 & 0.07 \\
\hline 868 & 8.82 & 111.43 & 2.25 & 0.07 \\
\hline 870 & 8.82 & 111.43 & 2.43 & 0.07 \\
\hline 872 & 8.82 & 111.43 & 2.13 & 0.06 \\
\hline 874 & 8.82 & 111.43 & 1.99 & 0.05 \\
\hline 876 & 8.82 & 111.43 & 2.36 & 0.06 \\
\hline 878 & 8.82 & 111.43 & 2.52 & 0.06 \\
\hline 880 & 8.82 & 111.43 & 2.20 & 0.05 \\
\hline 882 & 8.82 & 111.43 & 2.08 & 0.05 \\
\hline 884 & 8.82 & 111.43 & 2.07 & 0.03 \\
\hline 886 & 8.82 & 111.43 & 2.11 & 0.04 \\
\hline 888 & 8.82 & 111.43 & 2.19 & 0.05 \\
\hline 890 & 8.82 & 111.43 & 1.74 & 0.05 \\
\hline 892 & 8.82 & 111.43 & 1.90 & 0.05 \\
\hline 894 & 8.82 & 111.43 & 1.95 & 0.05 \\
\hline 896 & 8.82 & 111.43 & 1.84 & 0.05 \\
\hline 898 & 8.82 & 111.43 & 2.04 & 0.06 \\
\hline
\end{tabular}




\begin{tabular}{|c|c|c|c|c|}
\hline 900 & 8.82 & 111.43 & 2.29 & 0.05 \\
\hline 902 & 8.82 & 111.43 & 1.96 & 0.05 \\
\hline 904 & 8.82 & 111.43 & 2.41 & 0.04 \\
\hline 906 & 8.82 & 111.43 & 2.74 & 0.04 \\
\hline 908 & 8.82 & 111.43 & 2.83 & 0.04 \\
\hline 910 & 8.82 & 111.43 & 2.63 & 0.04 \\
\hline 912 & 8.82 & 111.43 & 2.26 & 0.04 \\
\hline 914 & 8.82 & 111.43 & 2.50 & 0.04 \\
\hline 916 & 8.82 & 111.43 & 2.69 & 0.03 \\
\hline 918 & 8.82 & 111.43 & 2.91 & 0.04 \\
\hline 920 & 8.82 & 111.43 & 2.58 & 0.04 \\
\hline 922 & 8.82 & 111.43 & 2.76 & 0.05 \\
\hline 924 & 8.82 & 111.43 & 3.02 & 0.05 \\
\hline 926 & 8.82 & 111.43 & 2.58 & 0.04 \\
\hline 928 & 8.82 & 111.43 & 2.86 & 0.04 \\
\hline 930 & 8.82 & 111.43 & 2.55 & 0.05 \\
\hline 932 & 8.82 & 111.43 & 2.77 & 0.05 \\
\hline 934 & 8.82 & 111.43 & 2.86 & 0.05 \\
\hline 936 & 8.82 & 111.43 & 2.85 & 0.04 \\
\hline 938 & 8.82 & 111.43 & 2.69 & 0.05 \\
\hline 940 & 8.82 & 111.43 & 2.87 & 0.04 \\
\hline 942 & 8.82 & 111.43 & 2.80 & 0.04 \\
\hline 944 & 8.82 & 111.43 & 2.77 & 0.04 \\
\hline 946 & 8.82 & 111.43 & 2.71 & 0.04 \\
\hline 948 & 8.82 & 111.43 & 2.80 & 0.04 \\
\hline 950 & 8.82 & 111.43 & 2.76 & 0.04 \\
\hline 952 & 8.82 & 111.43 & 2.81 & 0.05 \\
\hline 954 & 8.82 & 111.43 & 2.81 & 0.04 \\
\hline 956 & 8.82 & 111.43 & 2.92 & 0.04 \\
\hline 958 & 8.82 & 111.43 & 2.95 & 0.04 \\
\hline 960 & 8.82 & 111.43 & 2.83 & 0.04 \\
\hline 962 & 8.82 & 111.43 & 2.78 & 0.05 \\
\hline 964 & 8.82 & 111.43 & 2.76 & 0.04 \\
\hline 966 & 8.82 & 111.43 & 2.66 & 0.04 \\
\hline 968 & 8.82 & 111.43 & 2.96 & 0.04 \\
\hline 970 & 8.82 & 111.43 & 2.52 & 0.04 \\
\hline 972 & 8.82 & 111.43 & 2.79 & 0.04 \\
\hline 974 & 8.82 & 111.43 & 2.66 & 0.04 \\
\hline 976 & 8.82 & 111.43 & 2.64 & 0.04 \\
\hline 978 & 8.82 & 111.43 & 2.35 & 0.04 \\
\hline 980 & 8.82 & 111.43 & 2.45 & 0.04 \\
\hline 982 & 8.82 & 111.43 & 2.46 & 0.05 \\
\hline 984 & 8.82 & 111.43 & 2.81 & 0.05 \\
\hline 986 & 8.82 & 111.43 & 2.23 & 0.03 \\
\hline 988 & 8.82 & 111.43 & 3.00 & 0.05 \\
\hline 990 & 8.82 & 111.43 & 3.07 & 0.06 \\
\hline 992 & 8.82 & 111.43 & 2.93 & 0.05 \\
\hline 994 & 8.82 & 111.43 & 2.69 & 0.05 \\
\hline 996 & 8.82 & 111.43 & 2.67 & 0.05 \\
\hline 998 & 8.82 & 111.43 & 2.68 & 0.06 \\
\hline 1000 & 8.82 & 111.43 & 2.76 & 0.05 \\
\hline 1002 & 8.82 & 111.43 & 2.63 & 0.04 \\
\hline 1004 & 8.82 & 111.43 & 2.56 & 0.05 \\
\hline 1006 & 8.82 & 111.43 & 2.43 & 0.06 \\
\hline
\end{tabular}




\begin{tabular}{|c|c|c|c|c|}
\hline 1008 & 8.82 & 111.43 & 2.29 & 0.05 \\
\hline 1010 & 8.82 & 111.43 & 2.30 & 0.06 \\
\hline 1012 & 8.82 & 111.43 & 2.21 & 0.06 \\
\hline 1014 & 8.82 & 111.43 & 1.94 & 0.07 \\
\hline 1016 & 8.82 & 111.43 & 1.76 & 0.07 \\
\hline 1018 & 8.82 & 111.43 & 1.80 & 0.07 \\
\hline 1020 & 8.82 & 111.43 & 1.70 & 0.08 \\
\hline 1022 & 8.82 & 111.43 & 1.84 & 0.08 \\
\hline 1024 & 8.82 & 111.43 & 1.67 & 0.09 \\
\hline 1026 & 8.82 & 111.43 & 1.65 & 0.10 \\
\hline 1028 & 8.82 & 111.43 & 1.53 & 0.11 \\
\hline 1030 & 8.82 & 111.43 & 1.53 & 0.10 \\
\hline 1032 & 8.82 & 111.43 & 1.57 & 0.09 \\
\hline 1034 & 8.82 & 111.43 & 1.52 & 0.10 \\
\hline 1036 & 8.82 & 111.43 & 1.38 & 0.10 \\
\hline 1038 & 8.82 & 111.43 & 1.39 & 0.11 \\
\hline 1040 & 8.82 & 111.43 & 1.57 & 0.12 \\
\hline 1042 & 8.82 & 111.43 & 1.41 & 0.13 \\
\hline 1044 & 8.82 & 111.43 & 1.57 & 0.11 \\
\hline 1046 & 8.82 & 111.43 & 1.45 & 0.11 \\
\hline 1048 & 8.82 & 111.43 & 1.39 & 0.11 \\
\hline 1050 & 8.82 & 111.43 & 1.44 & 0.12 \\
\hline 1052 & 8.82 & 111.43 & 1.65 & 0.10 \\
\hline 1054 & 8.82 & 111.43 & 1.49 & 0.10 \\
\hline 1056 & 8.82 & 111.43 & 1.45 & 0.08 \\
\hline 1058 & 8.82 & 111.43 & 1.43 & 0.11 \\
\hline 1060 & 8.82 & 111.43 & 1.23 & 0.12 \\
\hline 1062 & 8.82 & 111.43 & 1.24 & 0.10 \\
\hline 1064 & 8.82 & 111.43 & 1.22 & 0.11 \\
\hline 1066 & 8.82 & 111.43 & 1.18 & 0.12 \\
\hline 1068 & 8.82 & 111.43 & 1.27 & 0.11 \\
\hline 1070 & 8.82 & 111.43 & 1.19 & 0.11 \\
\hline 1072 & 8.82 & 111.43 & 1.15 & 0.09 \\
\hline 1074 & 8.82 & 111.43 & 1.17 & 0.11 \\
\hline 1076 & 8.82 & 111.43 & 1.18 & 0.11 \\
\hline 1078 & 8.82 & 111.43 & 1.24 & 0.12 \\
\hline 1080 & 8.82 & 111.43 & 1.07 & 0.15 \\
\hline 1082 & 8.82 & 111.43 & 1.14 & 0.15 \\
\hline 1084 & 8.82 & 111.43 & 1.22 & 0.11 \\
\hline 1086 & 8.82 & 111.43 & 1.30 & 0.10 \\
\hline 1088 & 8.82 & 111.43 & 1.29 & 0.10 \\
\hline 1090 & 8.82 & 111.43 & 1.35 & 0.09 \\
\hline 1092 & 8.82 & 111.43 & 1.29 & 0.10 \\
\hline 1094 & 8.82 & 111.43 & 1.42 & 0.11 \\
\hline 1096 & 8.82 & 111.43 & 1.30 & 0.11 \\
\hline 1098 & 8.82 & 111.43 & 1.11 & 0.12 \\
\hline 1100 & 8.82 & 111.43 & 1.26 & 0.09 \\
\hline 1102 & 8.82 & 111.43 & 1.34 & 0.10 \\
\hline 1104 & 8.82 & 111.43 & 1.38 & 0.08 \\
\hline 1106 & 8.82 & 111.43 & 1.46 & 0.10 \\
\hline 1108 & 8.82 & 111.43 & 1.35 & 0.09 \\
\hline 1110 & 8.82 & 111.43 & 1.56 & 0.10 \\
\hline 1112 & 8.82 & 111.43 & 1.29 & 0.10 \\
\hline 1114 & 8.82 & 111.43 & 1.35 & 0.09 \\
\hline
\end{tabular}




\begin{tabular}{|c|c|c|c|c|}
\hline 1116 & 8.82 & 111.43 & 1.33 & 0.09 \\
\hline 1118 & 8.82 & 111.43 & 1.36 & 0.08 \\
\hline 1120 & 8.82 & 111.43 & 1.41 & 0.09 \\
\hline 1122 & 8.82 & 111.43 & 1.53 & 0.09 \\
\hline 1124 & 8.82 & 111.43 & 1.39 & 0.09 \\
\hline 1126 & 8.82 & 111.43 & 1.38 & 0.09 \\
\hline 1128 & 8.82 & 111.43 & 1.33 & 0.09 \\
\hline 1130 & 8.82 & 111.43 & 1.34 & 0.09 \\
\hline 1132 & 8.82 & 111.43 & 1.49 & 0.08 \\
\hline 1134 & 8.82 & 111.43 & 1.45 & 0.08 \\
\hline 1136 & 8.82 & 111.43 & 1.38 & 0.08 \\
\hline 1138 & 8.82 & 111.43 & 1.56 & 0.08 \\
\hline 1140 & 8.82 & 111.43 & 1.47 & 0.08 \\
\hline 1142 & 8.82 & 111.43 & 1.62 & 0.08 \\
\hline 1144 & 8.82 & 111.43 & 1.56 & 0.07 \\
\hline 1146 & 8.82 & 111.43 & 1.58 & 0.07 \\
\hline 1148 & 8.82 & 111.43 & 1.59 & 0.08 \\
\hline 1150 & 8.82 & 111.43 & 1.77 & 0.09 \\
\hline 1152 & 8.82 & 111.43 & 1.65 & 0.09 \\
\hline 1154 & 8.82 & 111.43 & 1.45 & 0.08 \\
\hline 1156 & 8.82 & 111.43 & 1.64 & 0.08 \\
\hline 1158 & 8.82 & 111.43 & 1.61 & 0.09 \\
\hline 1160 & 8.82 & 111.43 & 1.58 & 0.09 \\
\hline 1162 & 8.82 & 111.43 & 1.54 & 0.09 \\
\hline 1164 & 8.82 & 111.43 & 1.73 & 0.10 \\
\hline 1166 & 8.82 & 111.43 & 1.54 & 0.09 \\
\hline 1168 & 8.82 & 111.43 & 1.45 & 0.10 \\
\hline 1170 & 8.82 & 111.43 & & \\
\hline 1172 & 8.82 & 111.43 & 1.45 & 0.10 \\
\hline 1174 & 8.82 & 111.43 & 1.46 & 0.10 \\
\hline 1176 & 8.82 & 111.43 & 1.32 & 0.10 \\
\hline 1178 & 8.82 & 111.43 & 1.45 & 0.11 \\
\hline 1180 & 8.82 & 111.43 & 1.52 & 0.10 \\
\hline 1182 & 8.82 & 111.43 & 1.52 & 0.10 \\
\hline 1184 & 8.82 & 111.43 & 1.26 & 0.09 \\
\hline 1186 & 8.82 & 111.43 & 1.48 & 0.11 \\
\hline 1188 & 8.82 & 111.43 & 1.40 & 0.09 \\
\hline 1190 & 8.82 & 111.43 & 1.47 & 0.11 \\
\hline 1192 & 8.82 & 111.43 & 1.30 & 0.12 \\
\hline 1194 & 8.82 & 111.43 & 1.29 & 0.13 \\
\hline 1196 & 8.82 & 111.43 & 1.50 & 0.13 \\
\hline 1198 & 8.82 & 111.43 & 1.36 & 0.12 \\
\hline 1200 & 8.82 & 111.43 & 1.40 & 0.12 \\
\hline 1202 & 8.82 & 111.43 & 1.28 & 0.09 \\
\hline 1204 & 8.82 & 111.43 & 1.46 & 0.11 \\
\hline 1206 & 8.82 & 111.43 & 1.44 & 0.11 \\
\hline 1208 & 8.82 & 111.43 & 1.40 & 0.11 \\
\hline 1210 & 8.82 & 111.43 & 1.47 & 0.13 \\
\hline 1212 & 8.82 & 111.43 & 1.46 & 0.12 \\
\hline 1214 & 8.82 & 111.43 & & \\
\hline 1216 & 8.82 & 111.43 & 1.43 & 0.11 \\
\hline 1218 & 8.82 & 111.43 & 1.51 & 0.11 \\
\hline 1220 & 8.82 & 111.43 & 1.56 & 0.09 \\
\hline 1222 & 8.82 & 111.43 & 1.47 & 0.09 \\
\hline
\end{tabular}




$\begin{array}{lllll}1224 & 8.82 & 111.43 & & \\ 1226 & 8.82 & 111.43 & 1.56 & 0.10 \\ 1228 & 8.82 & 111.43 & 1.63 & 0.10 \\ 1230 & 8.82 & 111.43 & 1.76 & 0.09 \\ 1232 & 8.82 & 111.43 & 4.24 & 0.08 \\ 1234 & 8.82 & 111.43 & 1.49 & 0.09 \\ 1236 & 8.82 & 111.43 & 1.60 & 0.10 \\ 1238 & 8.82 & 111.43 & 1.66 & 0.09 \\ 1240 & 8.82 & 111.43 & 1.79 & 0.09 \\ 1242 & 8.82 & 111.43 & 1.53 & 0.09 \\ 1244 & 8.82 & 111.43 & 1.49 & 0.11 \\ 1246 & 8.82 & 111.43 & 1.59 & 0.09 \\ 1248 & 8.82 & 111.43 & & \\ 1250 & 8.82 & 111.43 & 1.51 & 0.10 \\ 1252 & 8.82 & 111.43 & 1.40 & 0.11 \\ 1254 & 8.82 & 111.43 & 1.71 & 0.09 \\ 1256 & 8.82 & 111.43 & 1.56 & 0.10 \\ 1258 & 8.82 & 111.43 & 1.54 & 0.09 \\ 1260 & 8.82 & 111.43 & 1.73 & 0.09 \\ 1262 & 8.82 & 111.43 & 1.63 & 0.08 \\ 1264 & 8.82 & 111.43 & 5.13 & 0.11 \\ 1266 & 8.82 & 111.43 & 1.46 & 0.12 \\ 1268 & 8.82 & 111.43 & 1.41 & 0.10 \\ 1270 & 8.82 & 111.43 & 1.51 & 0.11 \\ 1272 & 8.82 & 111.43 & 1.31 & 0.13 \\ 1274 & 8.82 & 111.43 & 1.32 & 0.14 \\ 1276 & 8.82 & 111.43 & 1.25 & 0.11 \\ 1278 & 8.82 & 111.43 & 1.24 & 0.13 \\ 1280 & 8.82 & 111.43 & 1.33 & 0.12 \\ 1282 & 8.82 & 111.43 & 1.18 & 0.10 \\ 1284 & 8.82 & 111.43 & & \\ 1286 & 8.82 & 111.43 & 1.42 & 0.11 \\ 1288 & 8.82 & 111.43 & 1.18 & 0.10 \\ 1290 & 8.82 & 111.43 & 1.14 & 0.11 \\ 1292 & 8.82 & 111.43 & 1.26 & 0.10 \\ 1294 & 8.82 & 111.43 & 1.34 & 0.10 \\ 1296 & 8.82 & 111.43 & 1.42 & 0.10 \\ 1298 & 8.82 & 111.43 & 1.57 & 0.10 \\ 1300 & 8.82 & 111.43 & 1.47 & 0.08\end{array}$

High sea surface temperatures in tropical warm pools during the Pliocene http://www.nature.com/ngeo/jour

\begin{tabular}{|c|c|c|c|}
\hline 1143B 1H-3 & 9.37 & 113.28 & 1.71 \\
\hline 1143B 1H-4 & 9.37 & 113.28 & 1.07 \\
\hline 1143B 2H-1 & 9.37 & 113.28 & 2.04 \\
\hline 1143B 2H-2 & 9.37 & 113.28 & 0.66 \\
\hline 1143B 2H-2 & 9.37 & 113.28 & 1.21 \\
\hline 1143B 2H-5 & 9.37 & 113.28 & 3.26 \\
\hline $1143 \mathrm{C} 2 \mathrm{H}-3$ & 9.37 & 113.28 & 1.79 \\
\hline $1143 \mathrm{C} 2 \mathrm{H}-5$ & 9.37 & 113.28 & 2.28 \\
\hline 1143B 3H-3 & 9.37 & 113.28 & 3.36 \\
\hline 1143B 3H-4 & 9.37 & 113.28 & 2.19 \\
\hline 1143B 3H-5 & 9.37 & 113.28 & \\
\hline $1143 \mathrm{C} 3 \mathrm{H}-4$ & 9.37 & 113.28 & \\
\hline $1143 \mathrm{C} 3 \mathrm{H}-5$ & 9.37 & 113.28 & 2.34 \\
\hline
\end{tabular}




\begin{tabular}{|c|c|c|c|c|}
\hline 1143B 4H-3 & 9.37 & 113.28 & 2.10 & 0.17 \\
\hline 1143B 4H-4 & 9.37 & 113.28 & 3.41 & 0.13 \\
\hline 1143B 4H-5 & 9.37 & 113.28 & 4.32 & 0.12 \\
\hline 1143B 4H-6 & 9.37 & 113.28 & 2.33 & 0.30 \\
\hline $1143 \mathrm{~B} 5 \mathrm{H}-2$ & 9.37 & 113.28 & 1.10 & 0.20 \\
\hline $1143 \mathrm{~B} 5 \mathrm{H}-2$ & 9.37 & 113.28 & 2.48 & 0.16 \\
\hline 1143B 5H-3 & 9.37 & 113.28 & & \\
\hline 1143B 5H-5 & 9.37 & 113.28 & 2.26 & 0.10 \\
\hline 1143B 5H-6 & 9.37 & 113.28 & 0.69 & 0.27 \\
\hline $1143 \mathrm{C} 5 \mathrm{H}-5$ & 9.37 & 113.28 & 2.09 & 0.12 \\
\hline $1143 \mathrm{~B} 6 \mathrm{H}-1$ & 9.37 & 113.28 & 1.67 & 0.73 \\
\hline $1143 \mathrm{C} 6 \mathrm{H}-1$ & 9.37 & 113.28 & 3.56 & 0.25 \\
\hline $1143 \mathrm{C} 6 \mathrm{H}-2$ & 9.37 & 113.28 & 2.40 & 0.15 \\
\hline $1143 \mathrm{~B} 6 \mathrm{H}-4$ & 9.37 & 113.28 & 1.27 & 0.16 \\
\hline $1143 \mathrm{C} 6 \mathrm{H}-4$ & 9.37 & 113.28 & & \\
\hline $1143 \mathrm{C} 6 \mathrm{H}-6$ & 9.37 & 113.28 & 2.50 & 0.10 \\
\hline 1143B 7H-1 & 9.37 & 113.28 & 0.92 & 0.13 \\
\hline 1143B 7H-3 & 9.37 & 113.28 & 1.87 & 0.23 \\
\hline 1143B 7H-5 & 9.37 & 113.28 & 1.10 & 0.11 \\
\hline $1143 \mathrm{C} 7 \mathrm{H}-5$ & 9.37 & 113.28 & 2.08 & 0.08 \\
\hline $1143 \mathrm{C} 7 \mathrm{H}-6$ & 9.37 & 113.28 & 2.06 & 0.09 \\
\hline 1143B 8H-1 & 9.37 & 113.28 & 1.60 & 0.14 \\
\hline $1143 \mathrm{~B} 8 \mathrm{H}-2$ & 9.37 & 113.28 & & \\
\hline $1143 \mathrm{~B} 8 \mathrm{H}-3$ & 9.37 & 113.28 & 1.29 & 0.13 \\
\hline 1143B 8H-5 & 9.37 & 113.28 & 0.99 & 0.13 \\
\hline $1143 \mathrm{C} 8 \mathrm{H}-5$ & 9.37 & 113.28 & 1.14 & 0.10 \\
\hline 1143B 9H-1 & 9.37 & 113.28 & 1.43 & 0.23 \\
\hline 1143B 9H-2 & 9.37 & 113.28 & 2.15 & 0.15 \\
\hline 1143B 9H-4 & 9.37 & 113.28 & 1.67 & 0.21 \\
\hline 1143B 9H-5 & 9.37 & 113.28 & & \\
\hline 1143B 9H-6 & 9.37 & 113.28 & 1.98 & 0.14 \\
\hline 1143B 10H-1 & 19.37 & 113.28 & 1.86 & 0.08 \\
\hline $1143 \mathrm{~B} 10 \mathrm{H}-3$ & 9.37 & 113.28 & 2.35 & 0.12 \\
\hline $1143 \mathrm{C} 10 \mathrm{H}-3$ & 9.37 & 113.28 & 2.07 & 0.14 \\
\hline 1143B 10H-5 & $\$ 9.37$ & 113.28 & 1.12 & 0.17 \\
\hline 1143B 10H- 6 & 9.37 & 113.28 & 1.32 & 0.13 \\
\hline $1143 \mathrm{C} 10 \mathrm{H}-6$ & 99.37 & 113.28 & 0.98 & 0.13 \\
\hline 1143B $11 \mathrm{H}-1$ & 19.37 & 113.28 & 2.34 & 0.12 \\
\hline 1143B 11H-4 & 9.37 & 113.28 & 1.94 & 0.17 \\
\hline 1143B 11H-5 & 9.37 & 113.28 & & \\
\hline 1143B 11H- & 9.37 & 113.28 & 2.03 & 0.23 \\
\hline $1143 \mathrm{C} 11 \mathrm{H}-7$ & 9.37 & 113.28 & & \\
\hline 1143B $12 \mathrm{H}-3$ & 9.37 & 113.28 & 1.66 & 0.13 \\
\hline 1143B $12 \mathrm{H}-3$ & 9.37 & 113.28 & 2.15 & 0.17 \\
\hline 1143B 12H-4 & 9.37 & 113.28 & & \\
\hline 1143B 12H-6 & 99.37 & 113.28 & 2.05 & 0.15 \\
\hline $1143 \mathrm{C} 12 \mathrm{H}-6$ & 9.37 & 113.28 & 1.38 & 0.11 \\
\hline 1143B 13H-2 & 9.37 & 113.28 & 2.42 & 0.15 \\
\hline $1143 \mathrm{~B} 13 \mathrm{H}-4$ & 9.37 & 113.28 & & \\
\hline 1143B 13H-4 & 9.37 & 113.28 & 2.45 & 0.13 \\
\hline 1143B 13H-6 & 99.37 & 113.28 & & \\
\hline $1143 \mathrm{~A} 14 \mathrm{H}-3$ & 9.37 & 113.28 & & \\
\hline 1143B $14 \mathrm{H}-1$ & 9.37 & 113.28 & 2.00 & 0.16 \\
\hline $1143 \mathrm{C} 14 \mathrm{H}-1$ & 9.37 & 113.28 & 1.77 & 0.10 \\
\hline
\end{tabular}




\begin{tabular}{|c|c|c|c|c|}
\hline 1143B 14H-3 & 9.37 & 113.28 & 2.31 & 0.16 \\
\hline $1143 \mathrm{C} 14 \mathrm{H}-2$ & 9.37 & 113.28 & 2.04 & 0.07 \\
\hline $1143 \mathrm{C} 14 \mathrm{H}-3$ & 9.37 & 113.28 & & \\
\hline $1143 \mathrm{C} 14 \mathrm{H}-3$ & 9.37 & 113.28 & 2.04 & 0.10 \\
\hline $1143 \mathrm{C} 14 \mathrm{H}-4$ & 9.37 & 113.28 & & \\
\hline 1143B $14 \mathrm{H}-5$ & 9.37 & 113.28 & 2.32 & 0.11 \\
\hline $1143 \mathrm{C} 14 \mathrm{H}-6$ & 9.37 & 113.28 & 1.06 & 0.05 \\
\hline 1143B $15 \mathrm{H}-1$ & 9.37 & 113.28 & 1.24 & 0.17 \\
\hline 1143B $15 \mathrm{H}-2$ & 9.37 & 113.28 & 1.99 & 0.08 \\
\hline 1143B $15 \mathrm{H}-3$ & 9.37 & 113.28 & 1.94 & 0.11 \\
\hline 1143B $15 \mathrm{H}-4$ & 9.37 & 113.28 & & \\
\hline 1143B $15 \mathrm{H}-5$ & 9.37 & 113.28 & 1.57 & 0.10 \\
\hline $1143 \mathrm{~A} 16 \mathrm{H}-4$ & 9.37 & 113.28 & & \\
\hline 1143B $16 \mathrm{H}-1$ & 9.37 & 113.28 & 2.06 & 0.22 \\
\hline $1143 \mathrm{~A} 16 \mathrm{H}-4$ & 9.37 & 113.28 & & \\
\hline $1143 \mathrm{C} 16 \mathrm{H}-1$ & 9.37 & 113.28 & 1.04 & 0.17 \\
\hline 1143B $16 \mathrm{H}-3$ & 9.37 & 113.28 & 2.25 & 0.16 \\
\hline $1143 \mathrm{C} 16 \mathrm{H}-2$ & 9.37 & 113.28 & & \\
\hline 1143B $16 \mathrm{H}-5$ & 9.37 & 113.28 & 2.29 & 0.16 \\
\hline 1143B 16H- & 9.37 & 113.28 & 2.24 & 0.10 \\
\hline $1143 \mathrm{C} 16 \mathrm{H}-6$ & 9.37 & 113.28 & 2.21 & 0.11 \\
\hline $1143 \mathrm{C} 16 \mathrm{H}-6$ & 9.37 & 113.28 & & \\
\hline 1143B $17 \mathrm{H}-2$ & 9.37 & 113.28 & 1.96 & 0.22 \\
\hline 1143B $17 \mathrm{H}-3$ & 9.37 & 113.28 & & \\
\hline 1143B $17 \mathrm{H}-3$ & 9.37 & 113.28 & 1.71 & 0.35 \\
\hline 1143B $17 \mathrm{H}-4$ & 9.37 & 113.28 & 2.31 & 0.13 \\
\hline 1143B $17 \mathrm{H}-5$ & 9.37 & 113.28 & 2.15 & 0.20 \\
\hline 1143B 17H-6 & 9.37 & 113.28 & 2.24 & 0.11 \\
\hline $1143 \mathrm{C} 17 \mathrm{H}-5$ & 9.37 & 113.28 & & \\
\hline $1143 \mathrm{C} 17 \mathrm{H}-5$ & 9.37 & 113.28 & 2.01 & 0.15 \\
\hline $1143 \mathrm{~A} 18 \mathrm{H}-4$ & 9.37 & 113.28 & & \\
\hline 1143B $18 \mathrm{H}-3$ & 9.37 & 113.28 & 1.85 & 0.19 \\
\hline $1143 \mathrm{~B} 18 \mathrm{H}-3$ & 9.37 & 113.28 & 2.30 & 0.35 \\
\hline 1143A 18H-6 & 9.37 & 113.28 & & \\
\hline 1143B $18 \mathrm{H}-5$ & 9.37 & 113.28 & 2.52 & 0.13 \\
\hline 1143B 18H- 6 & 9.37 & 113.28 & 1.50 & 0.12 \\
\hline 1143B 18H- & 9.37 & 113.28 & 1.87 & 0.20 \\
\hline $1143 \mathrm{C} 18 \mathrm{H}-5$ & 9.37 & 113.28 & 1.87 & 0.17 \\
\hline $1143 \mathrm{C} 18 \mathrm{H}-6$ & 9.37 & 113.28 & & \\
\hline 1143B 19H-2 & 9.37 & 113.28 & 2.16 & 0.17 \\
\hline 1143B 19H-2 & 9.37 & 113.28 & & \\
\hline 1143B 19H-3 & 9.37 & 113.28 & 1.63 & 0.22 \\
\hline 1143B 19H-3 & 9.37 & 113.28 & 2.04 & 0.11 \\
\hline 1143B 19H-4 & 9.37 & 113.28 & 2.30 & 0.11 \\
\hline 1143B 19H-5 & 9.37 & 113.28 & & \\
\hline 1143B 19H-5 & 9.37 & 113.28 & 2.05 & 0.18 \\
\hline 1143B 19H-5 & 9.37 & 113.28 & 1.72 & 0.08 \\
\hline 1143B 20X-1 & 9.37 & 113.28 & 1.90 & 0.11 \\
\hline 1143B 20X-4 & 9.37 & 113.28 & 1.64 & 0.12 \\
\hline
\end{tabular}

Isoprenoid and branched GDGT-based proxies for surface sediments from marine, fjord and lake environments in (

$\begin{array}{lllll}\text { GeoB7118-1 } & -25.98 & -70.80 & 0.96 & 0.02 \\ \text { GeoB7122-2 } & -25.98 & -70.83 & 0.88 & 0.02 \\ \text { GeoB7129-1 } & -28.42 & -71.32 & 0.62 & 0.02\end{array}$




$\begin{array}{lllll}\text { GeoB7147-1 } & -31.97 & -71.67 & 0.57 & 0.02 \\ \text { GeoB7162-4 } & -36.53 & -73.67 & 0.67 & 0.02 \\ \text { GeoB7197-1 } & -40.98 & -74.55 & 0.85 & 0.02 \\ \text { GeoB7182-1 } & -44.15 & -75.15 & 1.25 & 0.06 \\ \text { MD07-3094 } & -44.15 & -75.15 & 1.01 & 0.03 \\ \text { GeoB7187-1 } & -44.28 & -75.38 & 0.95 & 0.03 \\ \text { GeoB7189-1 } & -44.32 & -75.37 & 0.93 & 0.02 \\ \text { MD07-3091 } & -44.32 & -75.37 & 0.95 & 0.04 \\ \text { MD07-3086 } & -48.45 & -76.27 & 0.92 & 0.02 \\ \text { MD07-3084 } & -49.17 & -76.57 & 1.02 & 0.02\end{array}$

GDGT distributions on the East Siberian Arctic Shelf: implications for organic carbon export, burial and de

\begin{tabular}{|c|c|c|c|}
\hline TB-17 & 72.29 & 132.92 & 0.66 \\
\hline TB-18 & 72.17 & 133.00 & 0.70 \\
\hline TB-19 & 72.09 & 132.78 & 0.66 \\
\hline TB-20 & 71.93 & 132.62 & 0.68 \\
\hline TB-22 & 71.88 & 132.11 & 0.79 \\
\hline TB-23 & 71.83 & 131.67 & 0.61 \\
\hline TB-24 & 71.76 & 131.17 & 0.63 \\
\hline TB-25 & 71.72 & 130.83 & 0.57 \\
\hline TB-26 & 71.69 & 130.58 & 0.74 \\
\hline TB-27 & 71.66 & 130.33 & 0.78 \\
\hline TB-28 & 71.62 & 130.04 & 0.86 \\
\hline TB-30 & 71.87 & 129.83 & 0.87 \\
\hline TB-31 & 71.86 & 130.32 & 0.72 \\
\hline TB-32 & 71.86 & 131.09 & 0.53 \\
\hline TB-33 & 72.09 & 131.09 & 0.59 \\
\hline TB-34 & 72.29 & 131.09 & 0.70 \\
\hline TB-35 & 72.46 & 131.09 & 0.59 \\
\hline TB-36 & 72.59 & 131.10 & 0.74 \\
\hline TB-37 & 72.71 & 131.09 & 0.77 \\
\hline TB-38 & 72.93 & 130.84 & 0.58 \\
\hline TB-39 & 72.93 & 130.66 & 0.67 \\
\hline TB-40 & 72.93 & 130.03 & 0.85 \\
\hline TB-43 & 72.89 & 131.93 & 0.57 \\
\hline TB-44 & 72.71 & 131.66 & 0.57 \\
\hline TB-45 & 72.70 & 130.66 & 0.70 \\
\hline TB-46 & 72.70 & 130.18 & 0.86 \\
\hline TB-47 & 72.45 & 130.12 & 0.87 \\
\hline TB-48 & 72.59 & 130.12 & 0.83 \\
\hline TB-49 & 72.59 & 130.68 & 0.64 \\
\hline TB-50 & 72.59 & 131.66 & 0.62 \\
\hline TB-52 & 72.45 & 130.67 & 0.68 \\
\hline TB-54 & 72.28 & 130.59 & 0.60 \\
\hline TB-55 & 72.29 & 131.72 & 0.55 \\
\hline TB-56 & 72.10 & 131.72 & 0.59 \\
\hline TB-57 & 72.00 & 131.77 & 0.65 \\
\hline TB-59 & 72.09 & 130.06 & 0.87 \\
\hline YS-9 & 73.37 & 130.00 & 0.60 \\
\hline YS-10 & 73.18 & 130.00 & 0.69 \\
\hline YS-11 & 73.02 & 129.99 & 0.79 \\
\hline YS-12B & 71.92 & 132.39 & 0.64 \\
\hline YS-13 & 71.97 & 131.70 & 0.60 \\
\hline YS-14 & 71.63 & 130.05 & 0.84 \\
\hline
\end{tabular}




\begin{tabular}{|c|c|c|c|c|}
\hline YS-15 & 71.63 & 130.05 & 0.86 & 0.76 \\
\hline YS-16 & 71.63 & 130.32 & 0.76 & 0.64 \\
\hline YS-17 & 71.63 & 130.19 & 0.81 & 0.66 \\
\hline YS-18 & 73.03 & 133.00 & 0.72 & 0.26 \\
\hline YS-19 & 73.04 & 133.46 & 0.63 & 0.35 \\
\hline YS-4 & 75.99 & 129.98 & 1.00 & 0.07 \\
\hline YS-5 & 75.27 & 130.02 & 0.85 & 0.09 \\
\hline YS-6 & 74.72 & 130.02 & 0.90 & 0.15 \\
\hline YS-28 & 72.65 & 154.19 & 0.77 & 0.15 \\
\hline YS-31 & 71.59 & 161.69 & 0.67 & 0.28 \\
\hline YS-36 & 69.82 & 166.00 & 0.77 & 0.12 \\
\hline YS-37 & 70.14 & 168.01 & 1.15 & 0.07 \\
\hline YS-38 & 70.70 & 169.13 & 1.01 & 0.05 \\
\hline YS-39 & 71.22 & 169.37 & 1.97 & 0.02 \\
\hline YS-40 & 71.48 & 170.55 & 1.87 & 0.02 \\
\hline YS-41 & 71.97 & 171.79 & 1.44 & 0.03 \\
\hline YS-86 & 75.30 & 174.40 & & 0.01 \\
\hline YS-88 & 75.10 & 172.19 & 2.14 & 0.01 \\
\hline YS-90 & 74.67 & 172.39 & 1.48 & 0.02 \\
\hline YS-91 & 74.43 & 170.85 & 1.58 & 0.02 \\
\hline YS-93 & 74.42 & 166.00 & 1.35 & 0.03 \\
\hline YS-95 & 74.42 & 161.34 & 1.10 & 0.04 \\
\hline YS-98 & 75.55 & 160.75 & 2.21 & 0.03 \\
\hline YS-99 & 75.17 & 163.59 & 1.07 & 0.06 \\
\hline YS-100 & 75.72 & 164.08 & 1.02 & 0.03 \\
\hline YS-102 & 76.56 & 160.07 & & 0.00 \\
\hline YS-104 & 76.93 & 155.17 & 1.03 & 0.05 \\
\hline YS-106 & 76.97 & 150.29 & 1.76 & 0.05 \\
\hline YS-111 & 75.00 & 160.01 & 1.15 & 0.06 \\
\hline YS-112 & 74.83 & 159.33 & 1.19 & 0.07 \\
\hline YS-116 & 74.58 & 157.00 & 0.78 & 0.07 \\
\hline YS-118 & 74.33 & 156.01 & 0.81 & 0.09 \\
\hline YS-120 & 73.29 & 155.17 & 0.82 & 0.09 \\
\hline YS-131 & 76.40 & 125.47 & 1.22 & 0.05 \\
\hline YS-8 & 73.57 & 130.01 & 0.92 & 0.10 \\
\hline YS-26 & 72.46 & 150.60 & 0.82 & 0.47 \\
\hline YS-27 & 72.57 & 152.37 & 0.72 & 0.28 \\
\hline YS-29 & 72.20 & 153.17 & 0.76 & 0.32 \\
\hline YS-30 & 71.36 & 152.15 & 0.82 & 0.58 \\
\hline YS-32 & 70.57 & 161.22 & 0.74 & 0.33 \\
\hline YS-33 & 70.17 & 161.22 & 0.92 & 0.30 \\
\hline YS-34B & 69.71 & 162.69 & 0.70 & 0.51 \\
\hline YS-35 & 69.82 & 164.06 & 0.66 & 0.28 \\
\hline YS-20 & 73.31 & 139.89 & 0.78 & 0.50 \\
\hline YS-21 & 73.09 & 140.35 & 0.94 & 0.46 \\
\hline YS-22 & 72.88 & 140.63 & 0.78 & 0.66 \\
\hline YS-22B & 72.89 & 140.62 & 0.85 & 0.59 \\
\hline YS-23 & 72.79 & 142.67 & 0.83 & 0.54 \\
\hline YS-24 & 73.05 & 142.67 & 0.83 & 0.56 \\
\hline YS-25 & 73.14 & 142.67 & 0.95 & 0.53 \\
\hline
\end{tabular}

Drastic changes in the distribution of branched tetraether lipids in suspended matter and sediments from th

$\begin{array}{lllll}\text { YM1 } & 70.86 & 83.43 & 1.03 & 0.98 \\ \text { YM2 } & 71.88 & 82.82 & 0.97 & 0.97\end{array}$




$\begin{array}{lllll}\text { YM3 } & 71.84 & 82.78 & 0.97 & 0.97 \\ \text { YM4 } & 71.81 & 82.75 & 1.03 & 0.97 \\ \text { YM5 } & 71.77 & 82.71 & 1.03 & 0.97 \\ \text { YG1 } & 72.65 & 80.14 & 0.81 & 0.75 \\ \text { YG2a } & 73.08 & 79.98 & 0.77 & 0.84 \\ \text { YG2b } & 73.08 & 79.98 & 0.70 & 0.58 \\ \text { YG3 } & 73.15 & 80.49 & 0.77 & 0.84 \\ \text { YG4 } & 73.36 & 80.49 & 0.70 & 0.83 \\ \text { YG5 } & 73.40 & 80.48 & 0.63 & 0.61 \\ \text { YG6 } & 72.72 & 79.11 & 0.76 & 0.80 \\ \text { YG7 } & 72.56 & 79.30 & 0.56 & 0.75 \\ \text { YG8 } & 72.45 & 77.05 & 0.77 & 0.67 \\ \text { YG9 } & 72.62 & 77.52 & 0.77 & 0.68 \\ \text { YO1 } & 74.29 & 78.62 & 0.59 & 0.65 \\ \text { YO2 } & 75.68 & 83.20 & 1.96 & 0.35 \\ \text { YO3 } & 76.09 & 84.86 & 0.95 & 0.22 \\ \text { KS1 } & 77.22 & 78.09 & 0.51 & 0.84 \\ \text { KS2 } & 75.84 & 68.91 & 0.54 & 0.75 \\ \text { KS3 } & 72.34 & 65.98 & 0.59 & 0.47 \\ \text { KS4 } & 78.48 & 72.80 & 0.53 & 0.53 \\ \text { KB1 } & 71.18 & 77.38 & 0.89 & 0.97 \\ \text { KB2 } & 71.19 & 77.46 & 0.97 & 0.97 \\ \text { KB3 } & 71.22 & 77.57 & 1.09 & 0.95 \\ \text { KB4 } & 71.24 & 77.69 & 0.95 & 0.97 \\ \text { KG1 } & 71.78 & 75.81 & 0.85 & 0.71 \\ \text { KG2 } & 71.78 & 75.98 & 0.88 & 0.71 \\ \text { OO1 } & 73.56 & 73.30 & 0.77 & 0.67 \\ \text { OO2 } & 73.84 & 75.09 & 0.67 & 0.73\end{array}$

Branched glycerol dialkyl glycerol tetraethers and crenarchaeol record post-glacial sea level rise and shift in

$\begin{array}{lllll}1 & 78.47 & 72.78 & 1.53 & 0.07 \\ 10 & 78.47 & 72.78 & 1.71 & 0.06 \\ 18 & 78.47 & 72.78 & 1.42 & 0.05 \\ 40 & 78.47 & 72.78 & 1.50 & 0.05 \\ 60 & 78.47 & 72.78 & 1.24 & 0.06 \\ 80 & 78.47 & 72.78 & 1.10 & 0.07 \\ 100 & 78.47 & 72.78 & 1.19 & 0.05 \\ 120 & 78.47 & 72.78 & 0.80 & 0.11 \\ 125 & 78.47 & 72.78 & 0.72 & 0.17 \\ 130 & 78.47 & 72.78 & 0.69 & 0.20 \\ 137 & 78.47 & 72.78 & 0.66 & 0.45 \\ 142 & 78.47 & 72.78 & 0.76 & 0.65 \\ 146 & 78.47 & 72.78 & 0.67 & 0.71 \\ 148 & 78.47 & 72.78 & 0.73 & 0.53 \\ 150 & 78.47 & 72.78 & 1.00 & 0.44 \\ 153 & 78.47 & 72.78 & 0.48 & 0.47 \\ 155 & 78.47 & 72.78 & 0.68 & 0.59 \\ 160 & 78.47 & 72.78 & 0.54 & 0.87 \\ 170 & 78.47 & 72.78 & 0.62 & 0.81 \\ 180 & 78.47 & 72.78 & 0.65 & 0.81 \\ 190 & 78.47 & 72.78 & 0.74 & 0.80 \\ 200 & 78.47 & 72.78 & 0.76 & 0.79 \\ 210 & 78.47 & 72.78 & 0.00 & 0.81 \\ 215 & 78.47 & 72.78 & 0.67 & 0.71\end{array}$




$\begin{array}{lllll}234 & 78.47 & 72.78 & 0.60 & 0.79 \\ 244 & 78.47 & 72.78 & 0.61 & 0.76 \\ 254 & 78.47 & 72.78 & 0.63 & 0.73 \\ 264 & 78.47 & 72.78 & 0.60 & 0.68 \\ 269 & 78.47 & 72.78 & 0.59 & 0.70 \\ 274 & 78.47 & 72.78 & 0.65 & 0.73 \\ 284 & 78.47 & 72.78 & 0.61 & 0.78 \\ 292 & 78.47 & 72.78 & 0.63 & 0.77\end{array}$

Constraints on the sources of branched tetraether membrane lipids in distal marine sediments http://dx.doi.

$\begin{array}{lllll}\text { GeoB2212-1 } & 4.03 & -25.62 & 4.37 & 0.06 \\ \text { GeoB2213-1 } & 1.27 & -24.15 & 4.87 & 0.01 \\ \text { GeoB2707-4 } & -41.95 & -56.32 & 0.34 & 0.01 \\ \text { GeoB2722-2 } & -47.33 & -58.62 & 0.40 & 0.00 \\ \text { GeoB2723-2 } & -48.91 & -57.88 & 0.57 & 0.02 \\ \text { GeoB2806-6 } & -37.83 & -53.14 & 0.60 & 0.01 \\ \text { GeoB2809-2 } & -36.33 & -51.52 & 0.52 & 0.02 \\ \text { GeoB2824-1 } & -33.50 & -42.50 & 2.66 & 0.01 \\ \text { GeoB6407-2 } & -42.04 & -19.50 & 3.53 & 0.01 \\ \text { GeoB6410-1 } & -44.52 & -20.90 & 5.44 & 0.01 \\ \text { GeoB8303-5 } & -34.26 & 16.78 & 0.70 & 0.01 \\ \text { GeoB8336-5 } & -29.21 & 12.34 & 2.44 & 0.01 \\ \text { GeoB8342-5 } & -31.50 & 13.00 & 2.80 & 0.01 \\ \text { GeoB9526-4 } & 12.43 & -18.06 & 0.55 & 0.02 \\ \text { GeoB9529-1 } & 8.35 & -17.37 & 0.33 & 0.02 \\ \text { IS-S2 } & 48.18 & -9.71 & 0.56 & 0.02 \\ \text { ENAM9407 } & 62.96 & -4.03 & 0.27 & 0.02 \\ \text { AII-GGC-22 } & -54.79 & -3.33 & & 0.03 \\ \text { T89-32 } & -14.97 & 10.67 & 1.49 & 0.07 \\ \text { T89-40 } & -21.62 & 6.78 & 2.67 & 0.01 \\ \text { NP-07-13-09 } & 79.07 & 10.67 & 1.77 & 0.02 \\ \text { NP-07-13-49 } & 79.01 & 11.38 & 1.67 & 0.01 \\ \text { HS 253 } & -75.00 & -26.00 & 3.81 & 0.02 \\ \text { GeoB10016--21.60 } & 96.66 & 0.27 & 0.02 \\ \text { GeoB10040-2 -6.48 } & 102.86 & 0.99 & 0.01 \\ \text { NIOP 902 } & 10.78 & 51.58 & 0.62 & 0.03 \\ \text { NIOP 903 } & 10.78 & 51.66 & 0.97 & 0.02 \\ \text { NIOP 904 } & 10.79 & 51.77 & 1.33 & 0.02 \\ \text { NIOP 907 } & 10.80 & 52.25 & & 0.01 \\ \text { NIOP 908 } & 10.78 & 52.92 & 1.64 & 0.02 \\ \text { Box 476 } & 24.10 & 65.47 & 2.42 & 0.03 \\ \text { PM1 } & -11.98 & -77.32 & 0.97 & 0.02 \\ \text { PM7 } & -11.05 & -78.07 & 1.89 & 0.03 \\ \text { F1-3c } & 30.00 & -123.99 & 0.61 & 0.07 \\ \text { F4-7c } & 27.38 & -123.33 & 1.24 & 0.07 \\ \text { Cariaco } & 10.67 & -65.60 & 0.46 & 0.01 \\ \text { MC-1 } & 41.30 & 141.55 & 1.07 & 0.02 \\ \text { BS } & 34.00 & 1.10 & 0.02\end{array}$

Core-top calibration of the lipid-based U $37 \mathrm{~K}^{\prime}$ mathContainer Loading Mathjax and TEX 86 temperatur

$\begin{array}{lllll}10701 & 40.00 & 17.47 & 267.53 & 0.03 \\ 10702 & 40.00 & 17.59 & 267.55 & 0.03 \\ 10703 & 40.00 & 17.74 & 267.58 & 0.04\end{array}$




\begin{tabular}{|c|c|c|c|c|}
\hline 10704 & 40.00 & 17.83 & 267.60 & 0.06 \\
\hline 10705 & 39.85 & 17.91 & 268.61 & 0.06 \\
\hline 10706 & 39.83 & 17.83 & & \\
\hline 10707 & 39.78 & 17.58 & 269.13 & 0.06 \\
\hline 10708 & 39.81 & 17.73 & 268.99 & 0.05 \\
\hline 10709 & 39.76 & 17.89 & 269.36 & 0.06 \\
\hline 10710 & 39.59 & 17.68 & 270.51 & 0.04 \\
\hline 10711 & 39.68 & 17.80 & 269.91 & 0.07 \\
\hline 10712 & 39.73 & 17.86 & 269.64 & 0.05 \\
\hline 10713 & 39.69 & 18.28 & 269.91 & 0.07 \\
\hline 10714 & 39.64 & 18.28 & 270.28 & 0.05 \\
\hline 10715 & 39.56 & 18.28 & 270.86 & 0.05 \\
\hline 10716 & 39.34 & 18.28 & 272.36 & 0.07 \\
\hline 10717 & 39.74 & 18.08 & 269.67 & 0.08 \\
\hline 10718 & 39.69 & 18.06 & 270.02 & 0.06 \\
\hline 10719 & 39.65 & 18.04 & 270.32 & 0.09 \\
\hline 10720 & 39.51 & 17.98 & 271.35 & 0.08 \\
\hline 10721 & 42.17 & 16.77 & 254.26 & 0.04 \\
\hline 10722 & 42.17 & 16.50 & 254.28 & 0.05 \\
\hline 10723 & 42.17 & 16.00 & 254.30 & 0.07 \\
\hline 10724 & 42.00 & 16.22 & 255.33 & 0.21 \\
\hline 10725 & 43.00 & 16.37 & 249.42 & 0.20 \\
\hline 10726 & 42.00 & 16.72 & 255.38 & 0.09 \\
\hline 10727 & 41.80 & 16.62 & 256.62 & 0.11 \\
\hline 10728 & 41.78 & 16.86 & 256.75 & 0.05 \\
\hline 10729 & 41.65 & 17.19 & 257.62 & 0.03 \\
\hline 10730 & 41.50 & 17.05 & 258.56 & 0.06 \\
\hline 10731 & 41.50 & 16.66 & 258.58 & 0.06 \\
\hline 10732 & 41.50 & 16.41 & 258.60 & 0.14 \\
\hline 10733 & 41.50 & 16.22 & 258.63 & 0.25 \\
\hline 10734 & 41.67 & 16.24 & 257.62 & 0.29 \\
\hline 10735 & 41.50 & 17.31 & 258.67 & 0.02 \\
\hline 10736 & 40.76 & 18.19 & 263.41 & 0.07 \\
\hline 10737 & 40.63 & 18.33 & 264.30 & 0.09 \\
\hline 10738 & 40.55 & 18.47 & 264.84 & 0.08 \\
\hline 10739 & 40.50 & 18.64 & 265.16 & 0.04 \\
\hline 10740 & 40.39 & 18.58 & 265.90 & 0.06 \\
\hline 10741 & 40.23 & 18.67 & 266.97 & 0.05 \\
\hline 10742 & 39.72 & 18.78 & 270.47 & 0.04 \\
\hline 10743 & 39.82 & 18.64 & 269.76 & 0.05 \\
\hline 10744 & 39.85 & 18.60 & 269.61 & 0.07 \\
\hline 10746 & 39.91 & 16.76 & 269.27 & 0.06 \\
\hline 10747 & 39.72 & 16.97 & 270.54 & 0.04 \\
\hline 10748 & 39.67 & 17.05 & 270.96 & 0.05 \\
\hline 10749 & 39.60 & 17.18 & 271.44 & 0.05 \\
\hline
\end{tabular}

Distribution of Crenarchaeota tetraether membrane lipids in surface sediments from the Red Sea

$\begin{array}{lllll}\text { MC359 } & 27.69 & 34.60 & 0.29 & 0.01 \\ \text { MC601 } & 27.71 & 35.05 & 0.27 & 0.03 \\ \text { MC596 } & 27.05 & 35.41 & 0.41 & 0.01 \\ \text { MC360 } & 26.29 & 35.36 & 0.29 & 0.07 \\ \text { MC362 } & 25.75 & 35.09 & 0.46 & 0.03\end{array}$




$\begin{array}{lllll}\text { MC363 } & 25.52 & 35.61 & 0.19 & 0.05 \\ \text { MC364 } & 24.76 & 36.23 & 0.32 & 0.05 \\ \text { MC365 } & 23.31 & 36.71 & 0.30 & 0.05 \\ \text { MC71 } & 23.39 & 36.98 & 0.18 & 0.07 \\ \text { MC73 } & 22.92 & 37.38 & 0.25 & 0.05 \\ \text { MC79 } & 22.25 & 37.78 & 0.27 & 0.09 \\ \text { MC85 } & 21.43 & 37.98 & 0.18 & 0.11 \\ \text { MC95 } & 19.91 & 38.01 & 0.89 & 0.03 \\ \text { MC101 } & 20.11 & 38.42 & 0.22 & 0.08 \\ \text { MC113 } & 19.64 & 38.61 & 0.17 & 0.07 \\ \text { MC111 } & 19.46 & 38.72 & 0.47 & 0.04 \\ \text { MC108 } & 19.14 & 39.05 & 0.51 & 0.05 \\ \text { MC105 } & 18.60 & 39.06 & 0.65 & 0.04 \\ \text { MC366 } & 17.36 & 40.02 & 0.68 & 0.03 \\ \text { MC367 } & 15.56 & 41.67 & 0.48 & 0.03 \\ \text { MC368 } & 12.40 & 44.49 & 0.78 & 0.04 \\ \text { MC120 } & 12.49 & 45.71 & 2.24 & 0.19 \\ \text { MC124 } & 12.87 & 47.42 & 2.46 & 0.05 \\ \text { MC126 } & 13.33 & 47.51 & 2.33 & 0.05 \\ \text { MC123 } & 13.08 & 47.92 & 1.26 & 0.09\end{array}$

Spatial distributions of core and intact glycerol dialkyl glycerol tetraethers (GDGTs) in the Columbia River

\begin{tabular}{lllll} 
CR St. 8 & 46.21 & -123.91 & 0.83 & 0.47 \\
\hline CR St. 9.1 & 46.27 & -124.01 & 0.74 & 0.71 \\
\hline CR St. 9.2 & 46.27 & -124.01 & 0.86 & 0.77 \\
\hline CR St. 9.3 & 46.27 & -124.01 & 0.80 & 0.72 \\
\hline CR St. 10 & 46.27 & -124.01 & 0.62 & 0.23 \\
\hline WB St. 1 & 46.60 & -123.90 & 0.65 & 0.45 \\
\hline WB St. 2 & 46.60 & -123.91 & 0.63 & 0.46 \\
\hline WB St. 3 & 46.61 & -123.92 & 0.60 & 0.38 \\
\hline WB St. 4 & 46.63 & -123.95 & 0.69 & 0.36 \\
\hline WB St. 6 & 46.69 & -123.73 & 0.64 & 0.72 \\
\hline WB St. 8 & 46.70 & -123.89 & 0.63 & 0.46 \\
\hline WB St. 9 & 46.71 & -123.85 & 0.60 & 0.56 \\
WB St. 10 & 46.67 & -123.81 & 0.63 & 0.65 \\
\hline WB St. 11 & 46.68 & -123.79 & 0.60 & 0.69 \\
\hline WB St. 12 & 46.71 & -123.85 & 0.58 & 0.55 \\
CR-15 & 46.17 & -124.33 & 0.98 & 0.04 \\
CR-40 & 46.17 & -124.91 & 1.39 & 0.01 \\
\hline CR-7 & 46.17 & -124.16 & 0.20 & 0.00
\end{tabular}

Constraints on the application of the MBT/CBT palaeothermometer at high latitude environments (Svalbari

$\begin{array}{llll}\text { NP-07-13-08 79.02 } & 10.73 & 1.67 & 0.19 \\ \text { NP-07-13-09 79.05 } & 10.65 & 1.77 & 0.18 \\ \text { NP-07-13-10 79.05 } & 10.83 & 2.06 & 0.16 \\ \text { NP-07-13-11 79.12 } & 11.65 & 1.25 & 0.04 \\ \text { NP-07-13-13 79.28 } & 11.67 & 1.00 & 0.01 \\ \text { NP-07-13-14 79.25 } & 11.67 & 1.00 & 0.01 \\ \text { NP-07-13-15 79.22 } & 11.70 & 1.00 & 0.02 \\ \text { NP-07-13-16 79.18 } & 11.75 & 1.14 & 0.06 \\ \text { NP-07-13-21 79.22 } & 11.92 & 0.67 & 0.03 \\ \text { NP-07-13-23 79.00 } & 11.02 & 2.00 & 0.16 \\ \text { NP-07-13-24 79.00 } & 10.85 & 1.89 & 0.15\end{array}$




$\begin{array}{llll}\text { NP-07-13-25 79.03 } & 10.87 & 1.63 & 0.19 \\ \text { NP-07-13-26 79.05 } & 11.08 & 1.81 & 0.14 \\ \text { NP-07-13-40 78.95 } & 11.95 & 1.22 & 0.07 \\ \text { NP-07-13-41 78.97 } & 11.78 & 1.58 & 0.11 \\ \text { NP-07-13-42 78.98 } & 11.83 & 1.33 & 0.08 \\ \text { NP-07-13-44 79.00 } & 11.72 & 1.33 & 0.08 \\ \text { NP-07-13-46 79.02 } & 11.48 & 1.44 & 0.08 \\ \text { NP-07-13-47 79.00 } & 11.60 & 1.43 & 0.06 \\ \text { NP-07-13-48 78.98 } & 11.50 & 1.67 & 0.11 \\ \text { NP-07-13-49 79.00 } & 11.37 & 1.67 & 0.11 \\ \text { NP-07-13-50 79.02 } & 11.30 & 1.57 & 0.12 \\ \text { NP-07-13-51 79.03 } & 11.37 & 1.73 & 0.20 \\ \text { NP-07-13-52 79.07 } & 11.37 & 1.33 & 0.03 \\ \text { NP-07-13-53 78.98 } & 11.65 & 1.36 & 0.10 \\ \text { NP-07-13-55 79.13 } & 11.73 & 1.00 & 0.06 \\ \text { NP-07-13-60 78.92 } & 12.05 & 1.00 & 0.03 \\ \text { NP-07-13-61 78.90 } & 12.40 & 1.00 & 0.01 \\ \text { NP-07-13-64 79.05 } & 11.48 & 1.57 & 0.07\end{array}$




\section{Global Soils}

Correlations between microbial tetraether lipids and environmental variables in Chinese soils: Optimizing the paleo-recons 1

Wudachi

DY-A

DY-1

DY-2

DY-3

DYLJ-1

DYLJ-3

XZ-49

CD-2

CD-5

CD-7

VSL-1

VSS-1

HS-S-2

HS-S-6

HS-S-11

WH2009-5-31

WH2009-1

WH2009-2

DHS-1

DHS-2

DHS-5

DHS-6

DHS-7

JFL-1a

JFL-2b

JFL-3

JFL-4b

JFL-5a

JFL-6a

JFL-7

JFL-8b

JFL-10a

JFL-1 1

JFL-12

JFL-13

JFL-14

JXZS-1

JXZS-2

JXZS-3

LP-1

LP-2

LP-3

LP-4

LP-5

LP-6

LP-7

LP-8

LP-9

LP-10

LP-11

LP-12

$$
37.75
$$

37.48

37.48

37.48

37.48

37.48

37.48

29.65

30.67

30.67

30.67

29.57

29.57

30.45

30.45

30.45

30.05

30.05

30.05

23.17

23.17

23.17

23.17

23.17

18.72

18.72

18.70

18.70

18.70

18.70

18.70

18.68

18.70

18.70

18.68

18.70

18.70

28.03

28.03

28.03

34.90

35.20

35.55

35.63

35.78

36.22

36.58

36.83

36.88

37.15

38.20

39.15
107.42

118.38

118.38

118.38

118.38

118.02

118.02

90.92

103.82

103.82

103.82

106.42

106.42

110.42

110.42

110.42

114.03

114.03

114.03

112.53

112.53

112.53

112.53

112.53

108.87

108.87

108.87

108.87

108.87

108.87

108.87

108.85

108.83

108.83

108.82

108.82

108.78

115.50

115.50

115.50

109.63

110.13

110.50

110.75

111.30

111.67

111.68

111.75

111.78

112.15

112.62

112.77
0.56

0.32

0.56

0.75

$0.39-0.62$

$0.35-0.31$

$0.38 \quad 0.28$

$0.33 \quad 0.98$

$0.20 \quad 0.94$

$0.12 \quad 0.88$

$0.33 \quad 0.75$

$0.15 \quad 0.84$

$0.31 \quad 0.73$

$0.47 \quad 0.93$

$0.53 \quad 0.91$

$0.47 \quad 0.90$

$0.07 \quad 0.98$

$0.10 \quad 0.98$

$0.08 \quad 0.95$

$0.21 \quad 0.95$

$0.07 \quad 0.96$

$0.00 \quad 0.98$

$0.05 \quad 0.92$

$0.04 \quad 0.97$

$0.06 \quad 0.99$

$0.07 \quad 1.00$

$0.08 \quad 1.00$

$0.06 \quad 1.00$

$0.05 \quad 0.99$

$0.07 \quad 1.00$

$0.07 \quad 1.00$

$0.09 \quad 0.99$

$0.05 \quad 0.98$

$0.08 \quad 0.97$

$0.15 \quad 0.99$

$0.10 \quad 0.96$

$0.08 \quad 0.91$

$0.14 \quad 0.97$

$0.14 \quad 0.99$

$0.09 \quad 0.97$

$0.48 \quad 0.69$

$0.57 \quad 0.81$

$0.50 \quad 0.68$

$0.43 \quad 0.42$

$0.36 \quad 0.35$

$0.53 \quad 0.30$

$0.48 \quad 0.42$

$0.53 \quad 0.55$

$0.50 \quad 0.28$

$0.49 \quad 0.56$

$0.65 \quad 0.54$

$0.56 \quad 0.57$ 


\begin{tabular}{|c|c|c|c|c|}
\hline LP-13 & 39.60 & 112.25 & 0.76 & 0.55 \\
\hline LP-14 & 39.90 & 111.77 & 0.66 & 0.83 \\
\hline LP-15 & 40.20 & 111.68 & 0.51 & 0.49 \\
\hline LP-16 & 39.65 & 111.87 & 0.66 & 0.48 \\
\hline LP-17 & 39.63 & 112.08 & 0.67 & 0.23 \\
\hline LP-18 & 39.02 & 112.35 & 0.67 & 0.65 \\
\hline LP-19 & 37.48 & 112.03 & 0.56 & 0.73 \\
\hline LP-20 & 37.25 & 111.63 & 0.73 & 0.72 \\
\hline LP-21 & 37.42 & 110.88 & 0.69 & 0.77 \\
\hline LP-22 & 37.57 & 110.50 & 0.76 & 0.84 \\
\hline LP-23 & 37.53 & 109.50 & 0.35 & 0.65 \\
\hline LP-24 & 37.60 & 108.83 & 0.57 & 0.53 \\
\hline LP-25 & 37.50 & 108.87 & 0.71 & 0.57 \\
\hline LP-26 & 37.35 & 108.93 & 0.59 & 0.59 \\
\hline LP-27 & 37.20 & 108.98 & 0.47 & 0.70 \\
\hline LP-28 & 36.97 & 109.25 & 0.64 & 0.68 \\
\hline LP-29 & 36.67 & 109.47 & 0.58 & 0.66 \\
\hline LP-30 & 36.40 & 109.50 & 0.64 & 0.75 \\
\hline LP-31 & 35.92 & 109.47 & 0.39 & 0.67 \\
\hline LP-32 & 35.72 & 109.33 & 0.51 & 0.83 \\
\hline LP-33 & 35.45 & 109.12 & 0.50 & 0.57 \\
\hline LP-34 & 35.22 & 109.03 & 0.54 & 0.66 \\
\hline Maoba-2 & 32.33 & 108.23 & 0.31 & 0.81 \\
\hline Maoba-1 & 32.30 & 108.20 & 0.48 & 0.43 \\
\hline PJ-4 & & & 0.41 & 0.64 \\
\hline PJ-6 & & & 0.41 & 0.93 \\
\hline Qinglingding & 33.83 & 108.80 & 0.44 & 0.94 \\
\hline TJ-1 & 38.70 & 117.52 & 0.55 & 0.72 \\
\hline TJ-3 & 38.73 & 117.47 & 0.71 & 0.71 \\
\hline TJ-6 & 38.70 & 117.43 & 0.46 & 0.46 \\
\hline TJ-9 & 38.70 & 117.45 & 0.61 & 0.78 \\
\hline XJBC-3 & 39.75 & 78.52 & 0.66 & 0.72 \\
\hline XJBC-5 & 39.75 & 78.52 & 0.74 & 0.74 \\
\hline Xunyangba-2 & 33.55 & 108.60 & 0.64 & 0.92 \\
\hline Yancheng & & & 0.33 & 0.47 \\
\hline Guangcheng & 32.43 & 108.32 & 0.21 & 0.98 \\
\hline 10NJS-1 & 34.35 & 109.53 & 0.41 & 0.63 \\
\hline 10NJS-2 & 34.35 & 109.53 & 0.40 & 0.72 \\
\hline 10NJS-3 & 34.35 & 109.53 & 0.39 & 0.75 \\
\hline 10NJS-4 & 34.35 & 109.53 & 0.41 & 0.75 \\
\hline 10NJS-5 & 34.35 & 109.53 & 0.33 & 0.77 \\
\hline DN-1 & 24.75 & 115.03 & 0.04 & 0.95 \\
\hline DN-2 & 24.75 & 115.03 & 0.06 & 0.90 \\
\hline DN-3 & 24.75 & 115.03 & 0.09 & 0.97 \\
\hline KM-1 & & & 0.09 & 0.98 \\
\hline KM-4 & 24.87 & 102.65 & 0.25 & 0.89 \\
\hline NN Moss-1 & 22.80 & 108.38 & 0.00 & 1.00 \\
\hline NN-1 & 22.80 & 108.38 & 0.05 & 0.89 \\
\hline NN-4 & 22.80 & 108.38 & 0.04 & 0.97 \\
\hline NN-6 & 22.78 & 108.37 & 0.03 & 1.00 \\
\hline TS-1 & 34.57 & 105.60 & 0.74 & 0.31 \\
\hline TS-3 & 34.57 & 105.60 & 0.62 & 0.58 \\
\hline TS-5 & 34.57 & 105.60 & 0.56 & 0.58 \\
\hline XN 1-0 & 36.52 & 101.87 & 0.97 & 0.50 \\
\hline
\end{tabular}




$\begin{array}{lllll}\text { XN 2-0 } & 36.52 & 101.87 & 1.02 & 0.28 \\ \text { XN 3-0 } & 36.52 & 101.87 & 0.78 & 0.45\end{array}$

Occurrence and distribution of tetraether membrane lipids in soils: Implications for the use of the TEX86 proxy and the BI'

\begin{tabular}{|c|c|c|c|c|}
\hline \multirow[t]{2}{*}{ Alaska-17 } & 64.87 & -147.83 & 0.52 & 0.66 \\
\hline & & & 0.58 & 0.90 \\
\hline \multirow[t]{2}{*}{ Cameroon-1 } & 4.23 & 9.33 & 0.11 & 0.82 \\
\hline & & & 0.00 & 0.98 \\
\hline \multirow[t]{4}{*}{ Canada-17 } & 49.95 & -98.18 & 0.54 & 0.73 \\
\hline & & & 0.58 & 0.74 \\
\hline & & & 0.59 & 0.89 \\
\hline & & & 0.69 & 0.88 \\
\hline \multirow[t]{4}{*}{ France-15 } & 45.05 & 2.55 & 0.23 & 0.94 \\
\hline & & & 0.17 & 0.92 \\
\hline & & & 0.20 & 0.96 \\
\hline & & & 0.17 & 0.95 \\
\hline \multirow[t]{3}{*}{ Gabon-1 } & 0.52 & 12.80 & 0.10 & 0.94 \\
\hline & & & 0.07 & 0.95 \\
\hline & & & 0.00 & 0.95 \\
\hline Gabon-2 & -1.52 & 14.12 & 0.00 & 0.96 \\
\hline \multirow[t]{2}{*}{ Gabon-3 } & -1.68 & 13.58 & 0.00 & 0.99 \\
\hline & & & 0.00 & 0.98 \\
\hline \multirow[t]{2}{*}{ Gabon-4 } & -2.22 & 11.53 & 0.00 & 0.97 \\
\hline & & & 0.00 & 1.00 \\
\hline Gabon-5 & -2.35 & 11.38 & 0.00 & 1.00 \\
\hline \multirow[t]{2}{*}{ Gabon-6 } & -0.52 & 10.28 & 0.08 & 0.74 \\
\hline & & & 0.00 & 0.92 \\
\hline \multirow[t]{2}{*}{ Ghana-2 } & 6.00 & 0.00 & 0.00 & 0.80 \\
\hline & & & & 0.84 \\
\hline Greece-13 & 40.50 & 23.55 & 0.21 & 0.98 \\
\hline \multirow[t]{2}{*}{ Greenland-05 } & 65.62 & 37.67 & 0.36 & 0.99 \\
\hline & & & 0.21 & 1.00 \\
\hline \multirow[t]{2}{*}{ Hawaii-10 } & 22.07 & -159.40 & 0.05 & 0.96 \\
\hline & & & 0.06 & 0.98 \\
\hline \multirow[t]{2}{*}{ Iceland-6 } & 65.35 & -20.88 & 0.30 & 0.99 \\
\hline & & & 0.37 & 0.98 \\
\hline \multirow[t]{2}{*}{ Ireland-9 } & 53.90 & -7.80 & 0.11 & 1.00 \\
\hline & & & 0.08 & 0.99 \\
\hline Italy-1 & 39.67 & 16.15 & 0.22 & 0.98 \\
\hline \multirow[t]{4}{*}{ Nigeria-15 } & 5.30 & 6.63 & 0.00 & 1.00 \\
\hline & & & 0.00 & 1.00 \\
\hline & & & 0.00 & 0.99 \\
\hline & & & 0.00 & 0.97 \\
\hline \multirow[t]{2}{*}{ Nigeria-19 } & 6.62 & 3.50 & 0.11 & 0.52 \\
\hline & & & 0.00 & 0.74 \\
\hline Scotland & 55.00 & -3.10 & 0.17 & 0.95 \\
\hline \multirow[t]{3}{*}{ South Africa-7 } & -29.78 & 30.68 & 0.00 & 0.97 \\
\hline & & & 0.00 & 0.99 \\
\hline & & & 0.00 & 0.95 \\
\hline \multirow[t]{2}{*}{ Spain-7 } & 38.98 & -6.33 & 0.17 & 0.95 \\
\hline & & & 0.11 & 0.90 \\
\hline \multirow[t]{2}{*}{ Sweden-4 } & 55.82 & 14.07 & 0.57 & 0.55 \\
\hline & & & 0.45 & 0.75 \\
\hline Texel-1 & 53.07 & 4.73 & 0.09 & 0.99 \\
\hline
\end{tabular}




$\begin{array}{lllll}\text { Texel-2 } & 53.07 & 4.73 & 0.07 & 1.00 \\ \text { Zaire-1 } & 0.87 & 24.47 & 0.07 & 0.95 \\ & & & 0.00 & 0.94 \\ & & & 0.00 & 0.94 \\ & & & 0.00 & 0.85 \\ \text { Zaire-2 } & 0.77 & 24.43 & 0.00 & 0.81 \\ & & & 0.00 & 0.99 \\ & & & \end{array}$

Distributions of isoprenoid and branched glycerol dialkanol diethers in Chinese surface soils and a loess-paleosol sequence

\begin{tabular}{|c|c|c|c|c|}
\hline 10GJ-1 & 34.35 & 109.53 & 0.35 & 0.44 \\
\hline 10GJ-9 & 34.35 & 109.53 & 0.36 & 0.50 \\
\hline 10GJ-16 & 34.35 & 109.53 & 0.29 & 0.58 \\
\hline 10GJ-24 & 34.35 & 109.53 & 0.25 & 0.77 \\
\hline 10GJ-31 & 34.35 & 109.53 & 0.26 & 0.75 \\
\hline 10GJ-41 & 34.35 & 109.53 & 0.28 & 0.67 \\
\hline $10 \mathrm{GJ}-48$ & 34.35 & 109.53 & 0.26 & 0.75 \\
\hline 10GJ-49 & 34.35 & 109.53 & 0.27 & 0.71 \\
\hline 10GJ-57 & 34.35 & 109.53 & 0.27 & 0.78 \\
\hline 10GJ-65 & 34.35 & 109.53 & 0.21 & 0.82 \\
\hline 10 YG-1 F2 & 34.35 & 109.53 & 0.21 & 0.82 \\
\hline $10 \mathrm{YG}-5 \mathrm{~F} 2$ & 34.35 & 109.53 & 0.28 & 0.78 \\
\hline $10 \mathrm{YG}-9 \mathrm{~F} 2$ & 34.35 & 109.53 & 0.23 & 0.86 \\
\hline 10 YG-13 F2 & 34.35 & 109.53 & 0.25 & 0.87 \\
\hline 10 YG-17 F2 & 34.35 & 109.53 & 0.25 & 0.84 \\
\hline $10 \mathrm{YG}-25 \mathrm{~F} 2$ & 34.35 & 109.53 & 0.27 & 0.83 \\
\hline 10 YG-29 F2 & 34.35 & 109.53 & 0.27 & 0.83 \\
\hline 11 YG-33 F2 & 34.35 & 109.53 & 0.24 & 0.88 \\
\hline 11 YG-37 F2 & 34.35 & 109.53 & 0.26 & 0.86 \\
\hline 10 YG-41 F2 & 34.35 & 109.53 & 0.29 & 0.84 \\
\hline 11 YG-45 F2 & 34.35 & 109.53 & 0.27 & 0.88 \\
\hline 11 YG-49 F2 & 34.35 & 109.53 & 0.30 & 0.89 \\
\hline $10 \mathrm{YG}-53 \mathrm{~F} 2$ & 34.35 & 109.53 & 0.32 & 0.89 \\
\hline 10 YG-57 F2 & 34.35 & 109.53 & 0.30 & 0.88 \\
\hline 10 YG-61 F2 & 34.35 & 109.53 & 0.29 & 0.91 \\
\hline 11 YG-65 F2 & 34.35 & 109.53 & 0.29 & 0.92 \\
\hline 10 YG-69 F2 & 34.35 & 109.53 & 0.31 & 0.91 \\
\hline 11 YG-73 F2 & 34.35 & 109.53 & 0.32 & 0.90 \\
\hline 11 YG-77 F2 & 34.35 & 109.53 & 0.31 & 0.91 \\
\hline 10 YG-81 F2 & 34.35 & 109.53 & 0.36 & 0.85 \\
\hline 11 YG-85 F2 & 34.35 & 109.53 & 0.38 & 0.81 \\
\hline 10 YG-89 F2 & 34.35 & 109.53 & 0.38 & 0.89 \\
\hline 10 YG-97 F2 & 34.35 & 109.53 & 0.36 & 0.81 \\
\hline $10 \mathrm{YG}-101 \mathrm{~F} 2$ & 34.35 & 109.53 & 0.37 & 0.88 \\
\hline $10 \mathrm{YG}-105 \mathrm{~F} 2$ & 34.35 & 109.53 & 0.37 & 0.90 \\
\hline $10 \mathrm{YG}-113 \mathrm{~F} 2$ & 34.35 & 109.53 & 0.36 & 0.91 \\
\hline $10 \mathrm{YG}-117 \mathrm{~F} 2$ & 34.35 & 109.53 & 0.35 & 0.87 \\
\hline $10 \mathrm{YG}-129 \mathrm{~F} 2$ & 34.35 & 109.53 & 0.31 & 0.90 \\
\hline $10 \mathrm{YG}-141 \mathrm{~F} 2$ & 34.35 & 109.53 & 0.31 & 0.91 \\
\hline $10 \mathrm{YG}-145 \mathrm{~F} 2$ & 34.35 & 109.53 & 0.33 & 0.87 \\
\hline $10 \mathrm{YG}-153 \mathrm{~F} 2$ & 34.35 & 109.53 & 0.30 & 0.87 \\
\hline $10 \mathrm{YG}-157 \mathrm{~F} 2$ & 34.35 & 109.53 & 0.32 & 0.88 \\
\hline 10 YG-165 F2 & 34.35 & 109.53 & 0.32 & 0.92 \\
\hline $10 \mathrm{YG}-177 \mathrm{~F} 2$ & 34.35 & 109.53 & 0.27 & 0.91 \\
\hline
\end{tabular}




$\begin{array}{lllll}10 \text { YG-181 F2 } & 34.35 & 109.53 & 0.29 & 0.92 \\ 10 \text { YG-197 F2 } & 34.35 & 109.53 & 0.33 & 0.92 \\ 10 \text { YG-201 F2 } & 34.35 & 109.53 & 0.29 & 0.92 \\ 10 \text { YG-205 F2 } & 34.35 & 109.53 & 0.27 & 0.93 \\ 10 \text { YG-213 F2 } & 34.35 & 109.53 & 0.28 & 0.92 \\ 10 \text { YG-221 F2 } & 34.35 & 109.53 & 0.28 & 0.95 \\ 10 \text { YG-225 F2 } & 34.35 & 109.53 & 0.32 & 0.91 \\ 11 \text { YG-233 F2 } & 34.35 & 109.53 & 0.30 & 0.91 \\ 12 \text { YG-237 F2 } & 34.35 & 109.53 & 0.29 & 0.93 \\ 10 \text { YG-245 F2 } & 34.35 & 109.53 & 0.32 & 0.91 \\ 10 \text { YG-249 F2 } & 34.35 & 109.53 & 0.33 & 0.89 \\ 10 \text { YG-261 F2 } & 34.35 & 109.53 & 0.27 & 0.93 \\ 10 \text { YG-265 F2 } & 34.35 & 109.53 & 0.32 & 0.90\end{array}$

Global calibration of a novel, branched GDGT-based soil pH proxy http://dx.doi.org/10.1016/j.orggeochem.2015.10.005

$\begin{array}{lllll}\text { xsc-150630 } & 47.10 & 119.89 & 0.18 & 0.97 \\ \text { xsc-150631 } & 34.16 & 95.90 & 0.89 & 0.91 \\ \text { xsc-150632 } & 37.02 & 100.80 & 0.96 & 0.54 \\ \text { xsc-150633 } & 36.69 & 101.30 & 0.99 & 0.64 \\ \text { xsc-150634 } & 46.05 & 121.79 & 0.19 & 0.76 \\ \text { xsc-150635 } & 29.25 & 91.68 & 0.42 & 0.67 \\ \text { xsc-150636 } & 33.77 & 95.66 & 0.64 & 0.82 \\ \text { xsc-150637 } & 35.55 & 102.03 & 0.72 & 0.63 \\ \text { xsc-150638 } & 37.74 & 108.91 & 0.50 & 0.61 \\ \text { xsc-150639 } & 39.87 & 111.18 & 0.37 & 0.85 \\ \text { xsc-150640 } & 49.94 & 121.43 & 0.24 & 0.95 \\ \text { xsc-150641 } & 53.29 & 122.15 & 0.26 & 1.00 \\ \text { xsc-150642 } & 32.09 & 92.27 & 0.51 & 0.85 \\ \text { xsc-150643 } & 40.45 & 113.19 & 0.40 & 0.63 \\ \text { xsc-150644 } & 29.06 & 90.39 & 0.50 & 0.94 \\ \text { xsc-150645 } & 39.33 & 111.19 & 0.39 & 0.62 \\ \text { xsc-150646 } & 49.33 & 120.97 & 0.53 & 0.78 \\ \text { xsc-150647 } & 29.33 & 88.98 & 0.54 & 0.70 \\ \text { xsc-150648 } & 36.02 & 105.88 & 0.73 & 0.55 \\ \text { xsc-150649 } & 35.78 & 104.05 & 0.82 & 0.71 \\ \text { xsc-150650 } & 35.78 & 104.05 & 0.81 & 0.72 \\ \text { xsc-150651 } & 29.29 & 91.15 & 0.55 & 0.65 \\ \text { xsc-150652 } & 43.44 & 110.08 & 0.42 & 0.70 \\ \text { xsc-150653 } & 44.61 & 120.97 & 0.30 & 0.61 \\ \text { xsc-150654 } & 38.84 & 110.44 & 0.41 & 0.55 \\ \text { xsc-150655 } & 35.27 & 100.64 & 0.85 & 0.75 \\ \text { xsc-150656 } & 38.04 & 109.24 & 0.48 & 0.39\end{array}$

Distribution of branched glycerol dialkyl glycerol tetraethers in surface soils of the Qinghai-Tibetan Plateau: implications c

$\begin{array}{lllll}\text { P756 } & 35.35 & 100.93 & 0.85 & 0.73 \\ \text { P760 } & 34.54 & 100.22 & 0.46 & 0.91 \\ \text { P765 } & 33.03 & 96.92 & 0.63 & 0.85 \\ \text { P773 } & 33.95 & 95.70 & 0.67 & 0.88 \\ \text { P775 } & 34.10 & 96.20 & 0.77 & 0.85 \\ \text { P782 } & 36.86 & 101.02 & 0.79 & 0.76 \\ \text { P787 } & 37.28 & 98.39 & 0.85 & 0.72 \\ \text { P796 } & 35.13 & 93.04 & 1.26 & 0.72 \\ \text { P801 } & 33.73 & 92.10 & 1.19 & 0.48 \\ \text { P802 } & 33.32 & 91.88 & 0.78 & 0.72\end{array}$




$\begin{array}{lllll}\text { P804 } & 32.19 & 91.68 & 0.96 & 0.86 \\ \text { P816 } & 29.28 & 90.64 & 0.34 & 0.98 \\ \text { P817 } & 29.19 & 90.62 & 0.23 & 0.98 \\ \text { P818 } & 29.20 & 90.62 & 0.24 & 0.98 \\ \text { P819 } & 29.21 & 90.64 & 0.25 & 0.95 \\ \text { P820 } & 29.22 & 90.63 & 0.34 & 0.96 \\ \text { P821 } & 29.23 & 90.63 & 0.32 & 0.78 \\ \text { P831 } & 28.24 & 88.77 & 0.78 & 0.99 \\ \text { P833 } & 28.26 & 87.79 & 0.58 & 0.93 \\ \text { P848 } & 29.55 & 84.64 & 0.78 & 0.83 \\ \text { P851 } & 29.44 & 85.67 & 0.75 & 0.81 \\ \text { P858 } & 29.90 & 90.13 & 0.59 & 0.94 \\ \text { P859 } & 29.89 & 90.12 & 0.35 & 0.98 \\ \text { P860 } & 29.87 & 90.12 & 0.34 & 0.99 \\ \text { P861 } & 29.84 & 90.06 & 0.33 & 0.97 \\ \text { P862 } & 29.80 & 90.03 & 0.28 & 1.00 \\ \text { P863 } & 29.71 & 89.96 & 0.38 & 0.94\end{array}$

Occurrence and distribution of extractable glycerol dialkyl glycerol tetraethers in podzols http://dx.doi.org/10.1016/j.orgger

$\begin{array}{lllll}\text { Litter } & 2.58 & 49.12 & 0.12 & 0.99 \\ 0-15 & 2.58 & 49.12 & 0.09 & 0.97 \\ & 2.58 & 49.12 & 0.06 & 1.00 \\ 15-30 & 2.58 & 49.12 & 0.05 & 1.00 \\ & 2.58 & 49.12 & 0.03 & 1.00 \\ 70-80 & 2.58 & 49.12 & 0.00 & 1.00 \\ & 2.58 & 49.12 & 0.04 & 1.00 \\ 80-90 & 2.58 & 49.12 & 0.03 & 1.00 \\ & 2.58 & 49.12 & 0.08 & 0.99 \\ \text { I AE } & 2.58 & 49.12 & 0.08 & 0.99 \\ & -63.00 & -2.00 & 0.00 & 1.00 \\ \text { II A12 } & -63.00 & -2.00 & & 1.00 \\ & -63.00 & -2.00 & 0.00 & 1.00 \\ \text { II Bhs } & -63.00 & -2.00 & & 1.00 \\ & -63.00 & -2.00 & 0.00 & 1.00 \\ \text { II Bh } & -63.00 & -2.00 & 0.00 & 1.00 \\ & -63.00 & -2.00 & 0.00 & 1.00 \\ \text { III A12 } & -63.00 & -2.00 & 0.21 & 0.99 \\ & -63.00 & -2.00 & 0.00 & 1.00 \\ & -63.00 & -2.00 & 0.00 & 1.00\end{array}$

Distributions of branched GDGTs in soils and lake sediments from western Uganda: Implications for a lacustrine paleother

$\begin{array}{lllll}1.00 & 30.01 & 0.33 & 0.05 & 0.98 \\ 2.00 & 30.00 & 0.33 & 0.05 & 0.97 \\ 3.00 & 29.99 & 0.33 & 0.05 & 0.99 \\ 4.00 & 29.98 & 0.33 & 0.05 & 1.00 \\ 8.00 & 29.97 & 0.33 & 0.08 & 0.99 \\ 7.00 & 29.96 & 0.33 & 0.11 & 0.96 \\ 9.00 & 29.95 & 0.33 & 0.20 & 0.97 \\ 5.00 & 29.94 & 0.33 & 0.10 & 0.97 \\ 6.00 & 29.93 & 0.33 & 0.13 & 0.94 \\ 10.00 & 29.92 & 0.33 & 0.43 & 0.96 \\ 11.00 & 29.91 & 0.33 & 0.42 & 0.95 \\ 12.00 & 29.90 & 0.33 & 0.71 & 0.98 \\ 14.00 & 29.89 & 0.33 & 0.82 & 0.99\end{array}$




\begin{tabular}{|c|c|c|c|c|}
\hline 15.00 & 29.88 & 0.33 & 0.54 & 0.91 \\
\hline 16.00 & 29.87 & 0.33 & 0.64 & 0.98 \\
\hline 24.00 & 29.86 & 0.33 & 0.66 & 0.96 \\
\hline 35.00 & 29.65 & 0.40 & 0.34 & 0.96 \\
\hline 37.00 & 29.65 & 0.39 & 0.33 & 0.99 \\
\hline 38.00 & 29.65 & 0.38 & 0.53 & 0.98 \\
\hline 25.00 & 29.65 & 0.37 & 0.67 & 0.99 \\
\hline $23 a$ & 29.65 & 0.36 & 0.25 & 1.00 \\
\hline 32.00 & 29.65 & 0.35 & 0.81 & 0.99 \\
\hline $23(b)$ & 29.65 & 0.34 & 0.77 & 1.00 \\
\hline 21.00 & 29.65 & 0.33 & 0.60 & 0.95 \\
\hline 30.00 & 29.65 & 0.32 & 0.44 & 0.93 \\
\hline 29.00 & 29.65 & 0.31 & 0.95 & 0.95 \\
\hline 17.00 & 29.65 & 0.30 & 1.23 & 0.92 \\
\hline 22.00 & 29.65 & 0.29 & 0.73 & 0.95 \\
\hline 18.00 & 29.65 & 0.28 & 0.52 & 0.95 \\
\hline 28.00 & 29.65 & 0.27 & 0.60 & 0.94 \\
\hline 19.00 & 29.65 & 0.26 & 0.42 & 0.98 \\
\hline 68.00 & 30.33 & 0.50 & 0.15 & 0.70 \\
\hline 50.00 & 30.33 & 0.50 & 0.19 & 0.62 \\
\hline 45.00 & 30.33 & 0.50 & 0.20 & 0.70 \\
\hline 42.00 & 30.33 & 0.50 & 0.22 & 0.63 \\
\hline 43.00 & 30.33 & 0.50 & 0.22 & 0.61 \\
\hline 48.00 & 30.33 & 0.50 & 0.13 & 0.77 \\
\hline 49.00 & 30.33 & 0.50 & 0.15 & 0.89 \\
\hline 44.00 & 30.33 & 0.50 & 0.16 & 0.85 \\
\hline 47.00 & 30.33 & 0.50 & 0.19 & 0.75 \\
\hline 57.00 & 30.33 & 0.50 & 0.26 & 0.71 \\
\hline 59.00 & 30.33 & 0.50 & 0.22 & 0.81 \\
\hline 56.00 & 30.33 & 0.50 & 0.23 & 0.76 \\
\hline 60.00 & 30.33 & 0.50 & 0.36 & 0.72 \\
\hline 61.00 & 30.20 & 0.45 & 0.35 & 0.75 \\
\hline 62.00 & 30.20 & 0.45 & 0.28 & 0.79 \\
\hline 41.00 & 30.20 & 0.45 & 0.07 & 0.95 \\
\hline 67.00 & 30.15 & 0.40 & 0.17 & 0.89 \\
\hline 65.00 & 30.15 & 0.40 & 0.24 & 0.79 \\
\hline 55.00 & 30.15 & 0.40 & 0.28 & 0.73 \\
\hline 54.00 & 30.15 & 0.40 & 0.31 & 0.93 \\
\hline 53.00 & 30.15 & 0.40 & 0.28 & 0.75 \\
\hline 52.00 & 30.25 & 0.66 & 0.29 & 0.84 \\
\hline 51.00 & 30.25 & 0.66 & 0.33 & 0.80 \\
\hline
\end{tabular}

Microbial glycerol dialkyl glycerol tetraethers from river water and soil near the Three Gorges Dam on the Yangtze River 1

$\begin{array}{lllll}\text { S-1 } & 110.97 & 30.87 & 0.12 & 0.92 \\ \text { S-2 } & 111.05 & 30.83 & 0.34 & 0.91 \\ \text { S-3 } & 111.06 & 30.83 & 0.00 & 0.91\end{array}$

Core and intact polar glycerol dialkyl glycerol tetraethers (GDGTs) in Sand Pond, Warwick, Rhode Island (USA): Insights

$\begin{array}{lllll}\text { SPS-1 } & 41.73 & -71.42 & 0.11 & 0.99 \\ \text { SPS-2 } & 41.73 & -71.42 & 0.08 & 0.98 \\ \text { SPS-3 } & 41.73 & -71.42 & 0.23 & 0.88 \\ \text { SPS-1 } & 41.73 & -71.42 & 0.11 & 0.96 \\ \text { SPS-2 } & 41.73 & -71.42 & 0.09 & 0.85 \\ \text { SPS-3 } & 41.73 & -71.42 & 0.22 & 0.75\end{array}$


Distribution of tetraether lipids in agricultural soils - differentiation between paddy and upland management

\begin{tabular}{|c|c|c|c|c|}
\hline Zeme & 45.18 & 8.67 & 0.14 & 0.85 \\
\hline Cixi & 30.22 & 121.35 & 0.23 & 0.63 \\
\hline Cixi & 30.18 & 121.35 & 0.22 & 0.84 \\
\hline Cixi & 30.10 & 121.50 & 0.19 & 0.81 \\
\hline Cixi & 30.18 & 121.08 & 0.22 & 0.61 \\
\hline Cixi & 30.17 & 121.13 & 0.13 & 0.82 \\
\hline Sukabumi & -6.87 & 106.93 & 0.01 & 0.91 \\
\hline Sukabumi & -6.87 & 106.93 & 0.01 & 0.91 \\
\hline Sukabumi & -6.87 & 106.93 & 0.01 & 0.94 \\
\hline Jasinga & -6.53 & 106.52 & 0.00 & 0.96 \\
\hline Jasinga & -6.53 & 106.52 & 0.00 & 0.97 \\
\hline Jasinga & -6.53 & 106.52 & 0.01 & 0.96 \\
\hline Ngawi & -7.43 & 111.60 & 0.00 & 0.89 \\
\hline Ngawi & -7.43 & 111.60 & 0.00 & 0.95 \\
\hline Ngawi & -7.43 & 111.60 & 0.00 & 0.93 \\
\hline Red Soil Station & 28.23 & 116.88 & 0.03 & 0.91 \\
\hline Red Soil Station & 28.23 & 116.88 & 0.02 & 0.92 \\
\hline Red Soil Station & 28.23 & 116.88 & 0.03 & 0.91 \\
\hline Laguna Los Banos & 14.23 & 121.34 & 0.00 & 0.97 \\
\hline Laguna Los Banos & 14.12 & 121.41 & 0.00 & 0.95 \\
\hline Laguna Los Banos & 14.21 & 121.34 & 0.00 & 0.99 \\
\hline Laguna Los Banos & 14.14 & 121.40 & 0.01 & 0.95 \\
\hline Laguna Los Banos & 14.19 & 121.37 & 0.01 & 0.96 \\
\hline Nueva Ecija & 15.67 & 120.84 & 0.00 & 0.97 \\
\hline Nueva Ecija & 15.60 & 120.91 & 0.01 & 0.87 \\
\hline Nueva Ecija & 15.58 & 120.91 & 0.01 & 0.80 \\
\hline Nueva Ecija & 15.62 & 120.95 & 0.01 & 0.92 \\
\hline Ifugao & 16.91 & 121.07 & 0.01 & 0.98 \\
\hline Ifugao & 16.91 & 121.13 & 0.01 & 0.99 \\
\hline Ifugao & 16.86 & 121.10 & 0.01 & 1.00 \\
\hline Ifugao & 16.86 & 121.10 & 0.01 & 0.98 \\
\hline Ifugao & 16.91 & 121.06 & 0.02 & 0.97 \\
\hline Hai Duong & 21.03 & 106.35 & 0.04 & 0.97 \\
\hline Hai Duong & 21.03 & 106.35 & 0.05 & 0.91 \\
\hline Vinh Phúc & 21.35 & 105.71 & 0.01 & 0.90 \\
\hline Vinh Phúc & 21.37 & 105.72 & 0.00 & 0.99 \\
\hline Vinh Phúc & 21.32 & 105.74 & 0.00 & 0.95 \\
\hline Cixi & 30.35 & 121.20 & 0.34 & 0.79 \\
\hline Cixi & 30.27 & 121.20 & 0.28 & 0.89 \\
\hline Cixi & 30.32 & 121.20 & 0.41 & 0.75 \\
\hline Sumbermujer & 8.13 & 113.00 & 0.00 & 0.99 \\
\hline Vinh Phúc & 21.35 & 105.70 & 0.00 & 0.99 \\
\hline Lào Cai & 22.30 & 103.89 & 0.01 & 0.99 \\
\hline Lào Cai & 22.41 & 103.90 & 0.00 & 0.99 \\
\hline Lào Cai & 22.40 & 103.84 & 0.00 & 0.99 \\
\hline Ifugao & 16.86 & 121.10 & 0.01 & 0.98 \\
\hline Ifugao & 16.91 & 121.07 & 0.01 & 0.99 \\
\hline Ifugao & 16.93 & 121.05 & 0.02 & 0.99 \\
\hline Vinh Phúc & 21.32 & 105.74 & 0.01 & 0.98 \\
\hline Lào Cai & 22.30 & 103.91 & 0.01 & 0.99 \\
\hline Lào Cai & 22.31 & 103.86 & 0.01 & 0.99 \\
\hline Cixi & 30.18 & 121.35 & 0.32 & 0.95 \\
\hline
\end{tabular}




\begin{tabular}{|c|c|c|c|c|}
\hline Cixi & 30.18 & 121.35 & 0.31 & 0.95 \\
\hline Cixi & 30.18 & 121.35 & 0.28 & 0.96 \\
\hline Cixi & 30.15 & 121.33 & 0.13 & 0.94 \\
\hline Cixi & 30.15 & 121.33 & 0.21 & 0.94 \\
\hline Cixi & 30.15 & 121.33 & 0.16 & 0.95 \\
\hline Cixi & 30.10 & 121.50 & 0.14 & 0.97 \\
\hline Cixi & 30.10 & 121.50 & 0.16 & 0.96 \\
\hline Cixi & 30.10 & 121.50 & 0.14 & 0.96 \\
\hline Cixi & 30.20 & 121.12 & 0.23 & 0.89 \\
\hline Cixi & 30.20 & 121.12 & 0.27 & 0.90 \\
\hline Cixi & 30.20 & 121.12 & 0.19 & 0.91 \\
\hline Cixi & 30.17 & 121.15 & 0.25 & 0.94 \\
\hline Cixi & 30.17 & 121.15 & 0.26 & 0.94 \\
\hline Cixi & 30.17 & 121.15 & 0.23 & 0.93 \\
\hline Cixi & 30.15 & 121.10 & 0.07 & 0.93 \\
\hline Cixi & 30.15 & 121.10 & 0.08 & 0.93 \\
\hline Cixi & 30.15 & 121.10 & 0.10 & 0.92 \\
\hline Cixi & 30.08 & 121.43 & 0.12 & 0.98 \\
\hline Cixi & 30.08 & 121.43 & 0.11 & 0.98 \\
\hline Cixi & 30.08 & 121.43 & 0.12 & 0.98 \\
\hline Sukabumi & -6.87 & 106.93 & 0.02 & 0.97 \\
\hline Sukabumi & -6.87 & 106.93 & 0.03 & 0.96 \\
\hline Sukabumi & -6.87 & 106.93 & 0.02 & 0.97 \\
\hline Jasinga & -6.53 & 106.52 & 0.01 & 1.00 \\
\hline Jasinga & -6.53 & 106.52 & 0.00 & 1.00 \\
\hline Jasinga & -6.53 & 106.52 & 0.00 & 1.00 \\
\hline Jasinga & -6.53 & 106.52 & 0.00 & 1.00 \\
\hline Ngawi & -7.43 & 111.60 & 0.03 & 0.94 \\
\hline Ngawi & -7.43 & 111.60 & 0.02 & 0.96 \\
\hline Ngawi & -7.45 & 111.60 & 0.02 & 0.96 \\
\hline Red Soil Station & 28.23 & 116.88 & 0.05 & 0.99 \\
\hline Red Soil Station & 28.23 & 116.88 & 0.04 & 0.98 \\
\hline Red Soil Station & 28.23 & 116.88 & 0.03 & 0.97 \\
\hline Red Soil Station & 28.23 & 116.88 & 0.03 & 0.97 \\
\hline Red Soil Station & 28.23 & 116.88 & 0.04 & 0.98 \\
\hline Padas village & -7.43 & 111.52 & 0.01 & 0.89 \\
\hline Sumbermujer & -8.13 & 113.02 & 0.03 & 0.97 \\
\hline Simo village & -7.48 & 111.60 & 0.02 & 0.86 \\
\hline Simo village & -7.12 & 111.60 & 0.04 & 0.85 \\
\hline Simo village & -7.12 & 111.60 & 0.06 & 0.86 \\
\hline Piladang & -0.25 & 100.58 & 0.03 & 0.99 \\
\hline Suntiang & -0.35 & 100.38 & 0.03 & 0.97 \\
\hline Suntiang & -0.35 & 100.38 & 0.02 & 0.96 \\
\hline Lukok & -0.37 & 100.40 & 0.02 & 0.95 \\
\hline Zeme & 45.18 & 8.67 & 0.14 & 0.98 \\
\hline Vercelli & 45.32 & 8.37 & 0.53 & 0.91 \\
\hline Vercelli & 45.32 & 8.37 & 0.72 & 0.94 \\
\hline Vercelli & 45.32 & 8.37 & 0.53 & 0.92 \\
\hline Vercelli & 45.32 & 8.37 & 0.18 & 0.98 \\
\hline Hai Duong & 10.43 & 106.05 & 0.01 & 0.99 \\
\hline Hai Duong & 10.43 & 106.05 & 0.01 & 1.00 \\
\hline Hai Duong & 10.38 & 106.05 & 0.01 & 0.97 \\
\hline Hai Duong & 10.38 & 106.05 & 0.01 & 0.97 \\
\hline Laguna Los Banos & 14.23 & 121.34 & 0.01 & 0.94 \\
\hline
\end{tabular}




\begin{tabular}{|c|c|c|c|c|}
\hline Laguna Los Banos & 14.23 & 121.34 & 0.02 & 0.99 \\
\hline Laguna Los Banos & 14.11 & 121.41 & 0.02 & 0.98 \\
\hline Laguna Los Banos & 14.12 & 121.41 & 0.01 & 0.97 \\
\hline Laguna Los Banos & 14.22 & 121.34 & 0.01 & 0.97 \\
\hline Laguna Los Banos & 14.22 & 121.34 & 0.02 & 0.98 \\
\hline Laguna Los Banos & 14.14 & 121.40 & 0.01 & 0.97 \\
\hline Laguna Los Banos & 14.14 & 121.40 & 0.01 & 0.97 \\
\hline Laguna Los Banos & 14.19 & 121.37 & 0.02 & 0.98 \\
\hline Laguna Los Banos & 14.19 & 121.36 & 0.01 & 0.99 \\
\hline Nueva Ecija & 15.67 & 120.84 & 0.02 & 0.99 \\
\hline Nueva Ecija & 15.67 & 120.84 & 0.02 & 0.99 \\
\hline Nueva Ecija & 15.67 & 120.88 & 0.01 & 0.98 \\
\hline Nueva Ecija & 15.66 & 120.88 & 0.02 & 0.99 \\
\hline Nueva Ecija & 15.67 & 120.92 & 0.02 & 0.99 \\
\hline Nueva Ecija & 15.67 & 120.92 & 0.02 & 1.00 \\
\hline Nueva Ecija & 15.60 & 120.91 & 0.01 & 0.87 \\
\hline Nueva Ecija & 15.60 & 120.91 & 0.03 & 0.98 \\
\hline Nueva Ecija & 15.61 & 120.95 & 0.02 & 0.98 \\
\hline Nueva Ecija & 15.62 & 120.95 & 0.03 & 0.99 \\
\hline Ifugao & 16.90 & 121.08 & 0.03 & 1.00 \\
\hline Ifugao & 16.91 & 121.13 & 0.02 & 1.00 \\
\hline Ifugao & 16.86 & 121.10 & 0.03 & 0.99 \\
\hline Ifugao & 16.91 & 121.06 & 0.06 & 0.99 \\
\hline Ifugao & 16.93 & 121.13 & 0.02 & 0.99 \\
\hline Ifugao & 16.86 & 121.10 & 0.05 & 1.00 \\
\hline Ifugao & 16.91 & 121.13 & 0.03 & 0.99 \\
\hline Ifugao & 16.92 & 121.06 & 0.08 & 1.00 \\
\hline Ifugao & 16.92 & 121.06 & 0.06 & 0.99 \\
\hline Ifugao & 16.93 & 121.14 & 0.04 & 0.99 \\
\hline Hai Duong & 21.03 & 106.35 & 0.05 & 0.99 \\
\hline Hai Duong & 21.03 & 106.35 & 0.05 & 0.99 \\
\hline Hai Duong & 20.99 & 106.41 & 0.05 & 0.99 \\
\hline Hai Duong & 20.99 & 106.41 & 0.07 & 0.96 \\
\hline Hai Duong & 20.96 & 106.44 & 0.04 & 0.99 \\
\hline Hai Duong & 20.96 & 106.44 & 0.05 & 0.99 \\
\hline Hai Duong & 20.95 & 106.36 & 0.08 & 0.99 \\
\hline Hai Duong & 20.94 & 106.37 & 0.05 & 0.98 \\
\hline Vinh Phúc & 21.35 & 105.71 & 0.03 & 0.98 \\
\hline Vinh Phúc & 21.35 & 105.71 & 0.03 & 0.98 \\
\hline Vinh Phúc & 21.37 & 105.72 & 0.02 & 0.96 \\
\hline Vinh Phúc & 21.37 & 105.72 & 0.01 & 0.94 \\
\hline Vinh Phúc & 21.32 & 105.74 & 0.02 & 0.98 \\
\hline Vinh Phúc & 21.32 & 105.74 & 0.03 & 0.98 \\
\hline Vinh Phúc & 21.31 & 105.74 & 0.04 & 0.98 \\
\hline Vinh Phúc & 21.32 & 105.73 & 0.01 & 0.93 \\
\hline Lào Cai & 22.41 & 103.90 & 0.02 & 0.97 \\
\hline Lào Cai & 22.41 & 103.90 & 0.01 & 0.97 \\
\hline Lào Cai & 22.30 & 103.91 & 0.05 & 0.98 \\
\hline Lào Cai & 22.30 & 103.91 & 0.11 & 0.99 \\
\hline Lào Cai & 22.30 & 103.89 & 0.05 & 0.99 \\
\hline Lào Cai & 22.30 & 103.89 & 0.09 & 1.00 \\
\hline Lào Cai & 22.31 & 103.86 & 0.08 & 0.99 \\
\hline Lào Cai & 22.32 & 103.86 & 0.04 & 0.98 \\
\hline Lào Cai & 22.39 & 103.84 & 0.03 & 0.99 \\
\hline
\end{tabular}




$\begin{array}{lllll}\text { Lào Cai } & 22.39 & 103.84 & 0.08 & 0.99 \\ \text { Tien Giang } & 10.44 & 106.06 & 0.01 & 0.99 \\ \text { Tien Giang } & 10.44 & 106.06 & 0.01 & 0.99 \\ \text { Tien Giang } & 10.40 & 106.10 & 0.01 & 0.99 \\ \text { Tien Giang } & 10.41 & 106.10 & 0.01 & 0.99 \\ \text { Tien Giang } & 10.37 & 106.13 & 0.01 & 0.99 \\ \text { Tien Giang } & 10.38 & 106.11 & 0.01 & 0.98 \\ \text { Tien Giang } & 10.38 & 106.11 & 0.02 & 0.97 \\ \text { Tien Giang } & 10.41 & 106.11 & 0.01 & 0.99 \\ \text { Tien Giang } & 10.41 & 106.11 & 0.01 & 0.99\end{array}$

Potential of GDGTs as a temperature proxy along an altitudinal transect at Mount Rungwe (Tanzania)

$\begin{array}{lllll}1.00 & -9.40 & 33.91 & 0.16 & 0.85 \\ 2.00 & -9.40 & 33.91 & 0.11 & 0.85 \\ 3.00 & -9.41 & 33.92 & 0.00 & 0.92 \\ 4.00 & -9.37 & 33.80 & 0.13 & 0.79 \\ 5.00 & -9.36 & 33.80 & 0.13 & 0.76 \\ 6.00 & -9.35 & 33.82 & 0.21 & 0.89 \\ 7.00 & -9.33 & 33.76 & 0.10 & 0.99 \\ 8.00 & -9.33 & 33.76 & 0.00 & 0.90 \\ 9.00 & -9.33 & 33.81 & 0.13 & 0.85 \\ 10.00 & -9.32 & 33.81 & 0.08 & 0.94 \\ 11.00 & -9.30 & 33.81 & 0.06 & 0.97 \\ 12.00 & -9.28 & 33.81 & 0.06 & 0.93 \\ 13.00 & -9.26 & 3.82 & 0.11 & 0.92 \\ 14.00 & -9.24 & 33.82 & 0.07 & 0.81 \\ 15.00 & -9.36 & 33.82 & 0.11 & 0.94 \\ 16.00 & -9.23 & 33.81 & 0.15 & 0.92 \\ 17.00 & -9.02 & 33.57 & 0.18 & 0.94 \\ 18.00 & -9.02 & 33.56 & 0.02 & 0.92 \\ 19.00 & -9.07 & 33.41 & 0.12 & 0.93 \\ 20.00 & -9.15 & 33.43 & 0.15 & 0.94 \\ 21.00 & -9.14 & 33.47 & 0.14 & 1.00\end{array}$

Constraints on the application of the MBT/CBT palaeothermometer at high latitude environments (Svalbard, Norway) http:

$\begin{array}{lllll}\text { NA1 } & 78.92 & 11.93 & 0.89 & 1.00 \\ \text { NA2 } & 78.92 & 11.92 & 0.25 & 0.98 \\ \text { NA3 } & 78.92 & 11.92 & & \\ \text { NA4 } & 78.92 & 11.93 & & \\ \text { MP1 } & 79.18 & 11.17 & 0.24 & 0.96 \\ \text { MP2 } & 79.18 & 11.18 & 0.83 & 0.79 \\ \text { MP3 } & 79.20 & 11.27 & 0.23 & 0.98 \\ \text { MP4 } & 79.20 & 11.30 & 0.62 & 0.95 \\ \text { MP5 } & 79.20 & 11.32 & 0.79 & 0.90 \\ \text { MP6 } & 79.20 & 11.32 & 0.86 & 0.86 \\ \text { LB1 } & 78.20 & 15.60 & 0.25 & 0.99 \\ \text { LB2 } & 78.20 & 15.62 & & \end{array}$

Seasonal variability in concentrations and fluxes of glycerol dialkyl glycerol tetraethers in Huguangyan Maar Lake, SE Chi

$\begin{array}{lllll}\text { S1 } & 21.14 & 110.27 & 0.11 & 0.99 \\ \text { S2 } & 21.14 & 110.27 & 0.05 & 0.97 \\ \text { S3 } & 21.14 & 110.27 & 0.05 & 1.00 \\ \text { S4 } & 21.14 & 110.28 & 0.09 & 0.89 \\ \text { S5 } & 21.14 & 110.28 & 0.06 & 0.90\end{array}$




$\begin{array}{lllll}\text { S6-1 } & 21.14 & 110.29 & 0.06 & 0.93 \\ \text { S6-2 } & 21.14 & 110.29 & 0.05 & 0.94 \\ \text { S6-3 } & 21.14 & 110.29 & 0.04 & 0.93 \\ \text { S6-4 } & 21.14 & 110.29 & 0.07 & 0.84 \\ \text { S7 } & 21.14 & 110.29 & 0.11 & 0.94 \\ \text { S8 } & 21.15 & 110.29 & 0.13 & 0.94 \\ \text { S9 } & 21.15 & 110.29 & 0.04 & 0.98 \\ \text { S10 } & 21.15 & 110.28 & 0.05 & 0.95 \\ \text { S11 } & 21.15 & 110.28 & 0.10 & 0.81 \\ \text { S12 } & 21.15 & 110.27 & 0.09 & 0.87 \\ \text { S13 } & 21.15 & 110.29 & 0.02 & 0.95\end{array}$

Spatial distributions of core and intact glycerol dialkyl glycerol tetraethers (GDGTs) in the Columbia River basin, Washing Hood River A

$\begin{array}{llll}45.70 & -121.50 \quad 0.37 & 0.96\end{array}$

Hood River AB $\quad 45.70 \quad-121.50 \quad 0.33 \quad 0.96$

$\begin{array}{llll}\text { Mt Hood A } & 45.33 & -121.66 & 0.44\end{array}$

$\begin{array}{lllll}\text { Twisp surface } & 48.32 & -120.30 & 0.60 & 0.76\end{array}$

$\begin{array}{lllll}\text { Young River A } & 46.07 & -123.79 & 0.21 & 0.95\end{array}$ 
tructions in semi-arid and arid regions http://dx.doi.org/10.1016/j.gca.2013.10.041 

$\Gamma$ index http://dx.doi.org/10.1016/j.orggeochem.2006.07.018 
$\because$ Implications for the degradation of tetraether lipids http://dx.doi.org/10.1016/j.orggeochem.2013.11.003 
of brGDGTs-based proxies in cold and dry regions 
schem.2009.10.007

\section{mometer}


Ittp://dx.doi.org/10.1016/j.orggeochem.2012.11.014

into the origin of lacustrine GDGTs http://dx.doi.org/10.1016/j.gca.2011.10.018 



//dx.doi.org/10.1016/j.orggeochem.2009.03.004

na: Implications for the applicability of the MBT-CBT paleotemperature proxy in lacustrine settings 
;ton: Insights into origin and implications for the BIT index 